\title{
Pricing Network Effects
}

\author{
ITAY P. FAINMESSER \\ Carey Business School and Department of Economics, The Johns Hopkins University \\ E-mail: Itay_Fainmesser@jhu.edu \\ ANDREA GALEOTTI \\ Department of Economics, University of Essex \\ E-mail: agaleo@essex.ac.uk \\ First version received month/year; final version accepted month/year (Eds.)
}

\begin{abstract}
The increase in the information that firms can collect or purchase about network effects across consumers motivates two important questions: How does a firm's pricing strategy react to detailed information on network effects? Are the availability and use of such information beneficial or detrimental to consumer surplus?

We develop a model in which a monopoly sells a network good and price discriminates based on information about consumers' influence and consumers' susceptibility to influence. The monopoly optimally offers consumers price discounts for their influence and charges price premia for their susceptibility; the price premia and the price discounts are simple functions of the pattern of network effects. We determine under which conditions, relative to uniform price, consumer surplus increases, and we characterize the value of information on network effects for the monopoly.
\end{abstract}

Key words: Network effects, consumption externalities, influence, monopoly, pricing, price discrimination, networks, value of information, degree distribution

JEL Codes: D42, D62, D85, L00, M21

\section{INTRODUCTION}

New technologies allow firms to collect and to purchase detailed information on network effects across consumers ${ }^{1}$ How firms incorporate this information in their marketing strategies, as well as its effect on consumer surplus and firms' profits, are important yet underexplored topics. As a concrete example, consider a product that has a collaborative component, such as membership in social club, the telephone, videoconference technology, an online game, a social network website, a file-sharing tool, or a creative collaborative software. A potential consumer decides whether and how much to consume of the product based on her expectations of the consumptions of other consumers with whom she expects to interact: friends, colleagues, collaborators, or competitors. Now consider a monopoly selling the product. Once it has access to precise information about "who influences whom," the monopoly can set different prices for different consumers in

1. Facebook, MySpace and Twitter allow firms to target marketing efforts based on consumers' demographic characteristics, as well as on information on users' social interactions. A number of new companies provide marketing services to firms based on information on network effects obtained by quantitative analyses of consumers' online behavior. For example, Klout uses a variety of social networking websites' data to create Klout user profiles that are assigned a score of influence, the socalled Klout score. The primary business model for Klout involves companies paying Klout for 'Perks' campaigns. A similar example is Vocalpoint, a marketing service operated by Procter\&Gamble. 
order to leverage network effects and earn larger profits. Some consumers may receive the product at a lower price or even for free. Other consumers, in contrast, may be exploited by the new monopoly pricing strategy.

Depending on the product of interest, network effects can take different forms. When choosing her consumption, an individual may be affected by the consumption of the entire population-global network effects - or by the consumption of a subset of the population-local network effects. Moreover, recent empirical studies reveal that consumers may be heterogeneous in terms of network effects. There are consumers, referred to as influential, whose behavior has a large impact on the behavior of others, and there are consumers, referred to as susceptible, who are particularly sensitive to others' behavior ${ }^{2}$ Our model incorporates this rich set of environments in a tractable framework. We model the level of consumers' influence by their in-degree and their level of susceptibility to influence by their out-degree. Under the interpretation of local network effects, the out-degree is the number of other consumers that influence a consumer, and the in-degree is the number of other consumers that the consumer influences. The pattern of network effects is summarized by the in- and out-degree distributions of an underlying network of interactions. We assume, similar to Galeotti et al. (2010) and Sundararajan (2007), that when making consumption decisions, consumers know only their own in-degree and out-degree, and are uncertain about the level of interactions of other consumers. Under the interpretation of global network effects, the underlying assumption is that each consumer cares about a weighted average consumption, where the weight that consumer $i$ places on $j$ 's consumption depends on the susceptibility of $i$ and the influence of $j$.

We consider a two-stage game. In the first stage, the monopoly chooses a price scheme. In the second stage, consumers observe the price scheme and make simultaneous consumption decisions, taking into account the resulting network effects. The ability to price discriminate depends on the monopoly's knowledge about the network effects across consumers. In the benchmark case, the monopoly knows only the distributions of influence and susceptibility. We then consider the case in which the monopoly has more detailed information: it knows the influence of each consumer, the susceptibility of each consumer, and, finally, both. We compare these different scenarios and assess how the information that the monopoly has about the pattern of network effects shapes the optimal pricing strategy, aggregate consumer surplus, and monopoly profits.

Our first result characterizes the monopoly's optimal pricing strategy. The monopoly subsidizes influential consumers in order to boost network effects, and it extracts rents from highly susceptible consumers. The optimal price can, indeed, be decomposed into three additive components. The first part is the price that the monopoly would charge if it had no information about network effects. The second part captures the price premium that consumers pay for their susceptibility. The third component captures the price discount that consumers receive for their influence on others' demand. Both the price premium and the price discount are increasing in the average level of network effects and in the dispersion of influence and of susceptibility across consumers.

2. The development of new methods to identify influential and susceptible consumers from large data sets is a very active area of research in the intersection between the areas of business/marketing and information systems engineering. We refer to Probst et al. (2013) for a recent survey of this literature. More recently, Bapna and Umyarov (2014) take a randomized-experiment approach and show that individuals with fewer friends are more influenced by each one of their friends in their product adoption decisions. Companies such as Google are also increasing their effort in developing algorithms to identify influential consumers, e.g., Green (2008). 
There are many examples of price discrimination on the degree of consumers' influence. In the case of conspicuous consumption goods, fashion goods, as well as social interaction goods, firms often seed the market, by offering free products to selected influential consumers. A classic example is celebrities receiving free expensive products. Other examples of price discrimination based on consumers' influence are Klout Perks programs. These programs allow companies to provide exclusive rewards, coupons and discounts to consumers based on their influence on other consumers. Often, the discount offered to a consumer is proportional to her influence e.g., online fashion retailer Gilt Groupe offers consumers, through a Klout Perks campaign, discounts that are proportional to consumers' Klout scores ${ }^{3}$ Another relevant example of price discrimination takes the form of referral rebates, which allow sellers to price discriminate based on consumers' levels of influence, even without knowing their levels of influence at the time of purchase.

Our second result is a characterization of how consumers' utilities and consumer surplus change when firms are given access to detailed information on network effects ${ }^{4}$ We view this exercise as providing guidance to competition authorities that may be concerned with the effects on consumer surplus and welfare of allowing firms to use the newly available information on network effects in product markets. This is a part of a more general debate about the extent to which law should facilitate informational privacy, given the technological developments leading private and state organizations to enjoy unprecedented abilities to collect personal data; see Froomkin (2000) 5

When we move from uniform price to price discrimination, some consumers are worse off and others better off. When the monopoly has information on consumers' susceptibility, highly susceptible consumers lose because they are charged a high price premium. When the monopoly has information on consumers' influence, consumers who are neither very influential nor very susceptible are worse off because they receive a small price discount and do not enjoy much of the benefit from increased network effects.

Despite the ambiguous effect of price discrimination on individual consumers' utilities, we can fully characterize its effects on aggregate consumer surplus. We show that moving from no discrimination to price discrimination based on the level of consumers' influence always increases consumer surplus. When we move from no discrimination to price discrimination on the level of susceptibility, consumer surplus increases if, and only if, the average level of network effects is sufficiently high ${ }^{6}$ Note that linear pricing and concave consumers' utility function imply that consumers' equilibrium utility is increasing and convex in equilibrium consumption. Hence, to understand how consumer surplus changes when we move from uniform price to price discrimination, we need to evaluate the change in the average level of consumption and in the dispersion of consumption across consumers.

3. Similar examples include SponsoredTweets, which connects firms to influential users of Twitter.com so that they can be paid to tweet about their products, and the Like, Link, Love program by American Express.

4. We compare consumers' utilities and consumer surplus in the case of uniform price with the case of price discrimination on consumers' influence and of price discrimination on consumers' susceptibility.

5. There is a current debate about whether antitrust authorities should refocus their present investigation of Google on how Google's control of large data sets about consumers' behavior may entrench monopoly power and harm consumers' welfare, e.g., Newman (2013).

6. These results are derived in a context where, for each agent, the consumption of others has positive externalities. There are relevant examples, such as smoking, in which there are negative externalities across consumers' consumption. We discuss this case in Section 4.1 
It is intuitive that when moving from no discrimination to price discrimination, either on in-degrees or out-degrees, the monopoly prices in a way that boosts network effects. Hence, average consumption increases, and this tends to increase consumer surplus. More subtle is the effect on the dispersion of consumption. Price discrimination based on susceptibility is akin to a traditional form of price discrimination absent of network effects - i.e., charging higher prices to consumers whose demand schedule is higher. Thus, relative to uniform price, price discrimination on susceptibility dampens consumption of high-demand consumers and, thus, it decreases the dispersion in equilibrium consumption. Because this tends to reduce consumer surplus, the introduction of price discrimination on out-degrees has two confounding effects on consumer surplus. In contrast, price discrimination on consumers' influence is orthogonal to consumers' demand schedules and, therefore, does not dampen the consumption of high-demand consumers, ceteris paribus. At the same time, discrimination on influence increases the consumption of influential consumers and, thus, increases network effects on all individuals, but more so on highly susceptible consumers. As a consequence, consumption becomes more dispersed across consumers.

Finally, we evaluate the value of information on network effects for the monopoly. We define the value of information on, say, consumers' influence, as the increase in monopoly profits obtained by moving from no discrimination to price discrimination on in-degrees. We show that the value of having information on consumers' influence and/or consumers' susceptibility increases with the average level of network effects (average consumers' degree) and with the dispersion of the in- and out-degree distributions. Furthermore, information on the level of influence is more valuable than information on the level of susceptibility if, and only if, influence across consumers is more dispersed than susceptibility across consumers. This formalizes a recurrent and important theme in marketing stressing the importance of strategies that take advantage of structural differentiation, see Krackhardt (1996).

There is now convincing evidence that the patterns and level of network effects across consumers differ across product markets. In some product markets, the average influence is much higher than in others, and such differences are, in part, explained by the demographic characteristics of the representative consumer of that particular market, see, e.g., Leskovec et al. (2007), Keller et al. (2007), and Bearden and Etzel (1982) 7 Our result provides clear empirical predictions on how firms' investment in acquiring information on network effects (or firms' willingness to pay for such information) depends on the specific distribution of the patterns of network effects; these predictions should help to guide future empirical work 8

Our paper relates to the classical literature on network effects and network industries initiated in the 1980s by Farrell and Saloner (1985) and Katz and Shapiro (1985). Our

7. Bearden and Etzel (1982) shows that the level of network effects among consumers increases with the "conspicuousness" of the product. The level of influence among consumers is also higher for products that are "consumed publicly." Leskovec et al. (2007) shows that the level of social interactions - how much buyers recommend their products - and the degree of network effects - how often recommendations lead to adoption - change greatly across product categories: high recommendation and moderate influence for DVDs; moderate recommendation and high influence for books and music; and very low recommendation and influence for videos. Keller et al. (2007) report that both the average level and the dispersion of influence, measured by tracking word-of-mouth communication, is correlated to some demographic: it is much higher among teenagers as compared to the average in the population at large.

8. Section 5 extends the result on the value of information to the empirically relevant case of correlation between the level of influence of a consumer and the level of susceptibility of a consumer, and it provides new predictions that can be also taken to the data. 
main interest is in understanding how the monopoly can exploit consumers' heterogeneity with respect to such network effects. The literature on optimal marketing strategies in the presence of social influence is a recent and active field of research in economics, marketing, and computer science. Most of the literature has focused on optimal advertising and seeding strategies (distributing, initially, products for free to key consumers), given an exogenous process of product diffusion, e.g., Galeotti and Goyal (2009), Campbell (2013), Goyal and Kearns (2012), Domingos and Richardson (2001), and Kempe et al. (2003).

Few papers have studied optimal pricing as marketing strategies in environments with network effects. Hartline et al. (2008) and Arthur et al. (2009) consider dynamic optimal pricing in a setting in which adoption is sequential and consumers are myopic. They show that the optimal dynamic pricing strategy is NP-hard to compute, and they develop a simple pricing strategy to illustrate the potential value of exploiting influences across consumers.

Bloch and Quérou (2013) and Candogan et al. (2012) are the closest studies to ours. In Candogan et al. (2012), consumers' preferences are, as in our model, quadratic in consumption and linear in externality. The monopoly and the consumers have, however, perfect knowledge of the pattern of network effects across consumers, which is modeled as a deterministic graph. After characterizing optimal pricing, Candogan et al. (2012) focus mainly on computability and what optimal prices look like if only a few prices can be offered. Bloch and Quérou (2013) study a pricing setting with linear utility function and private valuation, and they also model network effects as a deterministic graph that is commonly known to the monopoly and consumers. Their focus is on optimal pricing when externalities result from consumption and from aspiration-based reference pricing $[9$

Modeling network effects as a deterministic graph and the assumption that its structure is commonly known to the agents add substantial complexity in the derived optimal pricing strategy. This prevents the understanding, for example, of how the optimal use of information on network effects shapes monopoly profits and consumer surplus, and how such effects depend on network characteristics ${ }^{10}$ We complement Bloch and Quérou (2013) and Candogan et al. (2012) by developing a model that can address these effects. We focus on a random network and limit the information that consumers and the monopoly have about the network effects. Under these assumptions, we obtain a tractable closed-form solution of the optimal pricing strategy, and we relate aggregate statistics of the distribution of network effects to consumer surplus and monopoly profits ${ }^{11}$

9. It is interesting to note that, despite the different approaches to modeling local network effects, there are some recurring effects. In particular, in these models, the optimal pricing of the monopoly can be decomposed into three additive components: a fixed price independent of the network; a discount based on the network effects that a consumer confers on others; and a premium based on the network effects that the consumer derives from interaction with others. The mathematical connection between the model of Candogan et al. (2012) and Bloch and Quérou (2013), and our model, is presented in online Appendix I.

10. Earier work on peer effects by Ballester et al. (2006) employed a deterministic graph-theoretic framework and showed that an individual's consumption depends on her Bonacich centrality in the network of externalities. Bloch and Quérou (2013) and Candogan et al. (2012) extend this framework to include pricing, and show that the price to a consumer also depends on her Bonacich centrality in the network. There are no general results that map how adding or redistributing links in a network affects the Bonacich centralities of agents, and this is one of the reasons that the models of Bloch and Quérou (2013) and Candogan et al. (2012) are less amenable to performe comparative statics.

11. The questions asked in our paper are also related to a recent literature that studies optimal subsidies to improve coordination across agents. Bernstein and Winter $(2012)$ study subsidies inducing efficient coordination in a complete-information setting. Sákovics and Steiner (2012) adopt a global game methodology and study subsidies that, at minimal cost, attain a given likelihood of successful 


\section{MODEL}

There is a single monopoly that can produce any quantity of a divisible good at no cost, and a unit measure of potential consumers $N=[0,1]$. Consumers' behavior depends on the consumption of their peers, and these network effects can be heterogeneous across consumers. In the first stage of the game, the monopoly chooses a price scheme $\mathbf{p}=\left\{p_{i}\right\}_{i \in N}$. The ability of the monopoly to assign different prices to different consumers depends on its knowledge about the patterns of network effects. In the second stage, consumers observe the price scheme and make simultaneous consumption decisions, $\mathbf{x}=\left\{x_{i}\right\}_{i \in N}$, taking into account the resulting network effects. We now formalize the ingredients of our model: patterns of network effects, consumers' preferences, and different levels of network knowledge of the monopoly.

We parametrize network effects by assigning each consumer with her out-degree, $k \in D=\left\{0, \ldots, k^{\max }\right\}$, and in-degree, $l \in D$. A consumer with out-degree $k$ draws, after making her consumption decision, $k$ others, and a consumer with in-degree $l$ is drawn by $l$ others ${ }^{12} \mathcal{F}: D^{2} \rightarrow[0,1]$ denotes the joint probability distribution of indegrees and out-degrees; $P: D \rightarrow[0,1]$ and $H: D \rightarrow[0,1]$ are the marginal probability distributions of out-degrees and in-degrees, respectively. We assume that there is no correlation in the population between a consumer's in-degree and her out-degree and vice-versa; that is, $F(k, l)=P(k) H(l)$ for all $k, l \in D$. Section 5 studies the case with correlation. Consistency requires that the average out-degree equals the average indegree; i.e., $\sum P(k) k=\hat{k}=\sum H(l) l$. We refer to $\hat{k}$ as the average level of network effects. We assume that the different draws of a consumer, as well as the draws of different consumers, are independent of each other. Bayes rule implies that the probability that consumer $i$ is sampled by $l \geq 1$ consumers, conditional on being sampled once, is $\bar{H}(l)=\frac{1}{\hat{k}} H(l) l$.

Consumers have quadratic utility that exhibits network effects. For a given profile of consumption $\mathbf{x}=\left(x_{i}, \mathbf{x}_{-i}\right)$ and unit price $p_{i}$, the utility of consumer $i$ who benefits from interacting with a finite set of consumers $N_{i}$ (i.e., draws the set of consumers $N_{i}$ ) is

$$
u_{i}\left(x_{i}, \mathbf{x}_{-i}, p_{i}\right)=x_{i}-\frac{1}{2} x_{i}^{2}+\gamma \sum_{j \in N_{i}} x_{i} x_{j}-p_{i} x_{i},
$$

where $\gamma>0$ is the positive network externalities coefficient. We discuss the role of the quadratic formulation in Section 2.1. Since different draws of a consumer, as well as the draws of different consumers, are independent of each other, we have that, for given $\left(x_{i}, \mathbf{x}_{-i}\right)$, the expected utility to consumer $i$ with out-degree $k$ is

$$
U_{i}\left(x_{i}, \mathbf{x}_{-i}, p_{i}, k\right)=\left(1-p_{i}\right) x_{i}-\frac{1}{2} x_{i}^{2}+\gamma x_{i} k A\left(\mathbf{x}_{-i}\right),
$$

where

$$
A\left(\mathbf{x}_{-i}\right) \triangleq E\left[x_{j} \mid j \in N_{i}\right]
$$

is the average consumption of a randomly selected consumer's neighbor. Given $A\left(\mathbf{x}_{-i}\right)$, the consumption of consumer $i$ depends on her type only via her out-degree $k$ and the

coordination.

12. More precisely, following Galeotti and Goyal (2009), a consumer with out-degree $k$ draws $k$ other consumers according to an atomless weighted-uniform distribution on the unit interval, where the weights are determined by the in-degrees of the sampled consumers. Therefore, the unconditional probability distribution function assigns to a consumer with in-degree $l$ a density for being sampled that is $l$ times higher than it assigns for a consumer with in-degree 1 . 
price $p_{i}$. Therefore, for any pricing scheme for which the price $p_{i}$ depends, at most, on the out-degree and in-degree of consumer $i$, we can write the demand of a consumer with out-degree $k$ and in-degree $l$ as $x(k, l)$; and

$$
A\left(\mathbf{x}_{-i}\right)=\sum_{l \in D} \bar{H}(l) \sum_{k \in D} P(k) x(k, l) .
$$

Before solving the monopoly's problem, we study the consumption equilibrium induced by the consumers' decisions in the second stage of the game, given any pricing scheme chosen by the monopoly. The following result establishes conditions under which there exists a unique consumption equilibrium and characterizes equilibrium consumptions levels. Let $\bar{p}$ be the average price paid by a randomly selected consumer's neighbour, i.e., $\bar{p}=\sum_{l \in D} \bar{H}(l) \sum_{k} P(k) p(k, l)$.

Proposition 1. Let $\mathbf{p}$ be any price schedule set by the monopoly in the first stage of the game and specifying for each consumer $i$ a price per unit $p_{i} \in \mathbb{R}$.

1 If $\gamma k^{\max }<1$, then, in the consumption stage, there exists a unique Bayes-Nash equilibrium $\mathbf{x}^{*}$.

2 Assume that $\gamma k^{\max }<1$ and that, according to $\mathbf{p}$, any two consumers with identical in- and out-degrees are priced the same. Then, in the unique equilibrium of the consumption stage, any two consumers with identical in-and out-degrees make the same consumption decision. In particular, let $p(k, l)$ and $x(k, l)$ be the price and the demand of a consumer with out-degree $k$ and in-degree $l$. Then, in the unique equilibrium of the consumption stage

$$
x(k, l)=1-p(k, l)+\frac{\gamma(1-\bar{p})}{1-\gamma \hat{k}} k .
$$

Uniqueness of equilibrium in the consumption stage is obtained when consumption externalities are not too strong. Hereafter, we maintain the assumption that $\gamma k^{\max }<1$ and that consumers play the unique equilibrium in the consumption stage. In the first stage, the ability of the monopoly to design targeted pricing strategies depends on the information that the monopoly possesses about the network. In the benchmark case, the monopoly knows the distributions $P$ and $H$, but knows neither the in-degree nor the out-degree of any individual consumer. In such a situation, the monopoly charges a uniform price, i.e., $p(k, l)=p$ for all $k, l \in D$. We will compare this benchmark case with three other cases:

a. The monopoly knows the out-degree of each consumer and price discriminates on out-degree, i.e., $p(k, l)=p(k)$ for all $l \in D$.

b. The monopoly knows the in-degree of each consumer and price discriminates on in-degree, i.e., $p(k, l)=p(l)$ for all $k \in D$.

c. The monopoly knows the in-degree and the out-degree of each consumer and price discriminates on both.

In each of these cases, the monopoly chooses a price schedule $\{p(k, l)\}_{k, l \in D}$ to maximize its expected profit

$$
\Pi=\sum_{l \in D} \sum_{k \in D} H(l) P(k) x(k, l) p(k, l),
$$


where $x(k, l)$ is the equilibrium consumption decision given by expression (2.3), and the choice of $p(k, l)$ is restricted depending on the information that the monopoly possesses, as described above. The following assumption guarantees that the monopoly maximization problem is well-behaved and has a unique interior solution (see Proposition A11 in the Appendix). We maintain assumption 1 throughout the paper.

Assumption 1. $\gamma k^{\max }<\frac{1}{2}$.

\subsection{Interpretations of the model and discussion of assumptions}

Our main interpretation of the consumption stage is that when consumers make their consumption decisions, they take into account local network effects, but they have only partial information about their future interactions. An alternative interpretation is that network effects are global. In this case, our formulation posits that the marginal utility of each consumer depends on the weighted average consumption of the entire population of consumers, but with different intensities. For a formal connection between these two interpretations, see online Appendix I.

An important and novel element of our model is the distinction between the outdegree and the in-degree of a consumer. The out-degree parametrizes a consumer's susceptibility to influence: how many others does the consumer look up to, listen to, benefit from sharing the use of the good with. The in-degree parametrizes the influence of a consumer: how many other consumers look up to the consumer, listen to her, benefit from sharing the use of the good with her ${ }^{13}$ As discussed in the introduction, the separation between the level of influence and susceptibility of a consumer - as well as the possible correlations between these two dimensions - is important because it is empirically relevant in markets.

There are important applications - e.g., social interaction goods such as Facebook.com or online gaming, or collaboration tools such as Dropbox.com - for which it is natural to model consumers' interactions as symmetric. In our model, symmetric interaction is captured by a perfect correlation between consumers' in- and out-degrees. The case in which individuals have symmetric interactions, but some are more susceptible to influence than others, is captured by replacing an individual's out-degree $k$ with her "effective out-degree" $\widetilde{k}$, which equals $k$ times the relative susceptibility of that particular individual to be influenced by a single connection. A similar approach could be used to accommodate charismatic individuals who generate more influence in each of their interactions 14

We have adopted a quadratic utility function for consumers. Its mathematical appeal is that it induces linear best responses, and this allows to derive simple consumers' demand functions. Two observations are in order. First, our equilibrium analysis and results on optimal pricing strategy carry over to a more general class of utility functions

13. We use the expressions "influence" and "susceptibility to influence" because they are intuitive and have become standard terminology in the literature. We stress that the notion of influence in this paper is static and based on expectations of others' consumptions; so, our model abstracts entirely from dynamic aspects, which are clearly important when we think of processes of influence.

14. The richness of the model allows us to consider a variety of goods and patterns of network effects. In fact, we can easily accommodate situations in which an individual is influenced by the average consumption of her contacts, or by different moments of the distribution of her contacts' consumptionse.g., with goods that have a competitive element, such as online gaming, an individual wants to get to a level of expertise that is at least as high as the lowest/average/highest level of expertise of her contacts, or, alternatively, with goods that have comparable alternatives that also exhibit network effects, such as mobile phones brands, and other competing platforms. 
that induce linear best responses; see Bramoullé et al. (2014) for a discussion. Second, we note that, fixing the price, equilibrium consumer's consumption is increasing in her outdegree (see expression 2.3). The reason for this is that the consumption stage is a game of strategic complements, and it exhibits degree complementarities as defined by Galeotti et al. (2010) ${ }^{15}$ As Galeotti et al. (2010) show, this monotonicity of the equilibrium consumption choice with respect to out-degree is, in fact, obtained in a much more general environment. As we shall show, it is exactly because the demand of a consumer is increasing in her level of susceptibility that leads the monopoly to trade-off between subsidizing consumption of influential consumers and extracting rent from susceptible consumers. In this sense, the mechanism that our analysis builds on, is robust to other specifications of the consumers' utility function.

The specification of the utility function is such that the effect of peers' consumption on the consumer's level of utility and on the consumer's marginal utility are both positive; see expression 2.1. In Section 4.1 we discuss an alternative specification that incorporates negative consumption externalities.

We conclude by discussing our assumption that the monopoly can use only linear pricing strategies. Suppose that the monopoly observes the in-degree and out-degree of each consumer, and is able to employ non-linear pricing strategies. Then, it is optimal for the monopoly to compute the socially efficient consumption profile and to make take-itor-leave-it offers to consumers that leave each consumer with no rent. To be more specific, let $\sigma_{k}^{2}$ and $\sigma_{l}^{2}$ be the variance of the out-degree and in-degree distribution, respectively. Then, simple computations, provided in online Appendix III, reveal that the quantity offered to a consumer with in-degree $l$ and out-degree $k$ is:

$$
x(k, l)=1+\gamma \hat{k} \frac{\left[\hat{k}+\gamma \sigma_{l}^{2}\right] k+\left[\hat{k}+\gamma \sigma_{k}^{2}\right] l}{\hat{k}^{2}-2 \gamma \hat{k}^{3}-\gamma^{2} \sigma_{l}^{2} \sigma_{k}^{2}-\gamma^{2} \sigma_{l}^{2} \hat{k}^{2}-\gamma^{2} \sigma_{k}^{2} \hat{k}^{2}},
$$

and the corresponding price charged for that quantity is

$$
T(k, l)=x(k, l)-\frac{1}{2}(x(k, l))^{2}+\gamma x(k, l) k A\left(\mathbf{x}_{-i}\right) .
$$

In this useful benchmark case, the monopoly does not face the trade-offs of leaving rents to highly influential consumers in order to extract surplus from highly susceptible consumers; in fact, all consumers are left with no rent. These trade-offs surface, however, as soon as the monopoly faces some unobservable heterogeneity, in which case non-linear pricing strategies become less effective in extracting additional consumer surplus relative to the optimal linear pricing strategy. We illustrate this in the context of a simple binary product-adoption model.

Consider an indivisible good and consumers with unit demand for the good. Assume, further, that each consumer $i$ has an intrinsic value $\theta_{i}$ drawn independently from the uniform distribution over the unit interval and that $\theta_{i}$ is private information to consumer $i$. Beyond their intrinsic values, consumers also experience local network effects in the sense that their value for the good increases by the constant value $\gamma>0$ for each one of their neighbors who adopts the good. That is, the utility of consumer $i$ is zero if she does not adopt, and it is $\theta_{i}+\gamma s-p_{i}$ if she and $s$ of her neighbors adopt.

From the monopoly's perspective, since it does not observe consumers' intrinsic values, for any given price schedule, consumers' strategies are captured by their

15. Degree complementarity means that the marginal expected utility of consumption is increasing in the out-degree; it is immediate to see that expression 2.1 satisfies this condition. 
probabilities of adoptions $\left\{x_{i}\right\}_{i \in N}$ such that $x_{i} \in[0,1]$. To be more specific, given $\mathbf{x}_{-i}$, the monopoly knows that a consumer $i$ adopts the good with probability $1-p_{i}+\gamma \sum_{j \in N_{i}} x_{j}$. It then follows that the probability that a consumer $i$ with out-degree $k$ adopts the good is $x_{i}=1-p_{i}+\gamma k A\left(\mathbf{x}_{-i}\right)$. This is the first-order condition in the divisible good formulation (when $x_{i}$ is interpreted as a quantity). So, in the simple case of binary product adoption with unobserved heterogeneity about the value of the product for each consumer, the resulting non-linear pricing game is equivalent to the linear pricing game considered in this paper.

\section{OPTIMAL PRICING AND CONSUMPTION}

In our benchmark case the monopoly has information on the distributions of influence and susceptibility, $H$ and $P$, but has no information on consumers' types and, therefore, charges a uniform linear price, $p(k, l)=p$ for all $k, l \in D$.

Proposition 2. If the monopoly knows only the distributions $P$ and $H$, then the optimal price is $p=1 / 2$, and the equilibrium consumption level of a consumer with out-degree $k$ and in-degree $l$ is $x(k, l)=(1+\gamma k /(1-\gamma \hat{k})) / 2$. The expected monopoly profit is $\Pi=1 /(4(1-\gamma \hat{k}))$.

The price charged is independent of the characteristics of the network, and, therefore, the equilibrium demand depends only on her susceptibility. In particular, network effects imply that a consumer's demand is increasing in her out-degree. This effect is linear because of the quadratic specification (i.e., linear best reply). Constant price and increasing linear demand in out-degree, then, imply that the average equilibrium consumption and the monopoly profits are both increasing in the average level of network effects 16

\subsection{Optimal pricing}

We now derive the properties of the optimal pricing schemes, when the monopoly has additional information on individual consumers' types. Consider the case in which the monopoly price discriminates based on consumers' susceptibility; that is, $p(k, l)=p_{\text {out }}(k)$ for all $k, l \in D$. We write the monopoly profits as $\Pi_{\text {out }}=\sum_{k \in D} P(k) \pi(k)$, where $\pi(k)=p_{\text {out }}(k) x(k)$ is the profit from a consumer with a level of susceptibility $k$, and $x(k)$ is the equilibrium consumption decision given in Proposition 1. The derivative of $\Pi_{\text {out }}$ with respect to the price charged to consumers with out-degree $k$ is

$$
\frac{\partial \Pi_{\text {out }}}{\partial p_{\text {out }}(k)}=P(k)\left[x(k)-p_{\text {out }}(k)+\sum_{k^{\prime} \in D} P\left(k^{\prime}\right) \frac{\partial \pi\left(k^{\prime}\right)}{\partial \bar{p}}\right],
$$

16. That profit is increasing (and convex) in average influence is not surprising, because an increase in average influence has a component corresponding to an upward shift in the demand function. Perhaps more surprising is that only the average level of influence affects profits. In particular, in a setup without network effects, Johnson and Myatt (2006) show that an increase in the dispersion of types corresponds to a rotation of the demand function, which has a non-monotonic effect on monopoly profit. In our setting, because of the quadratic utility function formulation, type dispersion under uniform price does not generate such effects. This highlights that the effects of type dispersion under the richer price regimes considered below are due to price discrimination and not demand rotation à la Johnson and Myatt (2006) . 
where we recall that $\bar{p}$ is the expected average price paid by a (randomly chosen) consumer's neighbor. The first term is the marginal benefit of increasing $p_{\text {out }}(k)$. The sum of the second and the third terms is the marginal cost of raising $p_{\text {out }}(k)$. The first of these two terms is the marginal decrease in the demand of out-degree $k$ consumers, keeping constant the average peers' consumption. The third term is the decrease in monopoly profits through the change of peers' consumption: an increase in $p_{\text {out }}(k)$ increases the expected price that a consumer's neighbor pays, which, in turn, dampens peers' average consumption and, therefore, decreases consumers' demand.

Since consumers' demand is increasing in their level of susceptibility, the marginal benefit of increasing the price of consumers with level of susceptibility $k$ is increasing in $k$; the marginal cost is, in contrast, constant in consumers' susceptibility. Therefore, the monopoly charges, at the optimum, higher prices to higher out-degree consumers. To illustrate this further, note that by equating marginal costs with marginal benefits, we can write $p_{\text {out }}(k)$ in the following informative way:

$$
p_{\text {out }}(k)=\frac{1}{2}+\frac{1}{2} \gamma A(\mathbf{x}) k+\sum_{k^{\prime} \in D} P\left(k^{\prime}\right) \frac{\partial \pi\left(k^{\prime}\right)}{\partial \bar{p}} .
$$

The first term is the uniform price. The second term captures the price-premium that the consumer must pay because of her susceptibility to influence. This is the product of her level of susceptibility $k$, the parameter of network externalities $\gamma$ and the expected peers' consumption, $A(\mathbf{x})$, as defined in 2.2 The third term captures the price discount that the consumer receives because of her influence on her peers: the expected marginal decrease of the monopoly profits due to an increase in the average price expected by consumers' neighbors.

We can replicate this exercise for the case of price discrimination on consumers' influence, $p(k, l)=p_{i n}(l)$ for all $k, l \in D$, and obtain that the derivative of the monopoly profit $\Pi_{i n}$ with respect to the price charged to consumers with in-degree $l$ is

$$
\frac{\partial \Pi_{i n}}{\partial p_{i n}(l)}=H(l)\left[\sum_{k \in D} P(k) x(k, l)-p_{i n}(l)+\frac{l}{\hat{k}} \sum_{l^{\prime} \in D} \sum_{k \in D} H\left(l^{\prime}\right) P(k) \frac{\partial \pi(k, l)}{\partial \bar{p}}\right] .
$$

The interpretation of the three terms is analogous to the interpretation of the case of price discrimination on out-degrees. The key point to note here is that highly influential consumers contribute most to the average price paid by a consumer's neighbor, $\bar{p}=\sum_{l^{\prime} \in D} \bar{H}\left(l^{\prime}\right) p_{i n}\left(l^{\prime}\right)=\frac{1}{\hat{k}} \sum_{l^{\prime} \in D} H\left(l^{\prime}\right) l^{\prime} p_{i n}\left(l^{\prime}\right)$. Hence, the marginal costs of raising $p_{\text {in }}(l)$ are increasing in the in-degree $l$, and so, at the optimum, the price is decreasing in the level of consumers' influence.

The following proposition describes precisely how the price premium and the price discount depend on the information that the monopoly has, and the characteristics of the patterns of network effects, as captured by the average level of network effects, $\hat{k}$, and the variance of the in-degree and out-degree distributions-i.e., $\sigma_{l}^{2}$ and $\sigma_{k}^{2}$.

Proposition 3. The optimal price schedules for discrimination on out-degree $\left(p_{\text {out }}(k)\right)$, 
in-degree $\left(p_{\text {in }}(l)\right)$, and both $\left(p_{\text {in } / \text { out }}(k, l)\right)$, are the following:

$$
\begin{aligned}
p_{\text {out }}(k) & =\frac{1}{2}+\frac{\gamma k}{4-4 \gamma \hat{k}-\gamma^{2} \sigma_{k}^{2}}-\frac{\gamma}{2} \frac{\gamma \sigma_{k}^{2}+2 \hat{k}}{4-4 \gamma \hat{k}-\gamma^{2} \sigma_{k}^{2}}, \\
p_{\text {in }}(l) & =\frac{1}{2}+\frac{\gamma}{2} \frac{\gamma \sigma_{l}^{2}+2 \hat{k}}{4-4 \gamma \hat{k}-\gamma^{2} \sigma_{l}^{2}}-\frac{\gamma l}{4-4 \gamma \hat{k}-\gamma^{2} \sigma_{l}^{2}}, \\
p_{\text {in /out }}(k, l) & =\frac{1}{2}+\frac{\gamma \hat{k}}{2} \frac{\left(\gamma \sigma_{l}^{2}+2 \hat{k}\right) k-\left(\gamma \sigma_{k}^{2}+2 \hat{k}\right) l}{\hat{k}^{2}\left(4-4 \gamma \hat{k}-\gamma^{2}\left(\sigma_{k}^{2}+\sigma_{l}^{2}\right)\right)-\gamma^{2} \sigma_{k}^{2} \sigma_{l}^{2}} .
\end{aligned}
$$

Figure 1(a) illustrates the optimal price function when the monopoly discriminates on susceptibility and when it discriminates on influence. Regardless of the information used by the monopoly, the optimal price can be decomposed into three terms: the uniform price, $1 / 2$; a price premium; and a price discount. As noted in the introduction, this decomposition is consistent with the findings of related works, such as Candogan et al. (2012) and Bloch and Quérou (2013). When the monopoly price discriminates only on susceptibility, the price discount is constant across consumers (the lowest intercept in Figure 1(a), whereas the price premium is increasing in consumers' susceptibility. The increase in the price paid by a consumer per unit increase in susceptibility (the slope of $p_{\text {out }}(k)$ in Figure $\left.1(\mathrm{a})\right)$, is $p_{\text {out }}(k+1)-p_{\text {out }}(k)=\gamma /\left(4-4 \gamma \hat{k}-\gamma^{2} \sigma_{k}^{2}\right)$. When the monopoly price discriminates on the level of influence, the price premium is constant across consumers (the highest intercept in Figure 1(a)p, whereas the price discount is increasing in the consumers' influence. The decrease in price paid by a consumer per unit increase in her influence (the slope of $p_{i n}(l)$ in Figure $\left.1(\mathrm{a})\right)$ is $p_{i n}(l)-p_{i n}(l+1)=\gamma /\left(4-4 \gamma \hat{k}-\gamma^{2} \sigma_{l}^{2}\right)$. This analysis, together with the expressions in Proposition 3 teach us that if the monopoly price discriminates on susceptibility (resp. influence), both the price premium and the price discount are increasing in the average level of network effects and in the dispersion of the levels of susceptibility (resp. influence) across consumers. The following corollary captures the aggregate impact of these changes on final prices.

Corollary 1. If the monopoly price discriminates on out-degrees (resp. in-degrees), the price charged to a consumer with a given in-degree and out-degree is decreasing (resp. increasing) in the average level of network effects and in the variance of the out-degree distribution (resp. in-degree distribution).

The dashed lines in Figure 1(b) are the optimal price functions that result from an increase in $\hat{k}$ or an increase in $\sigma_{k}^{2}$ (resp. $\sigma_{l}^{2}$ ) in the case of price discrimination on susceptibility (resp. influence). We provide here the intuitions for the case of price discrimination on consumers' susceptibility. An increase in the average level of network effects $\hat{k}$ increases the demand of each consumer, ceteris paribus. Subsequently, consumers are exposed to more peers' consumption, and, therefore, the price premium per additional out-degree is higher-i.e., the slope of the optimal price function $p_{\text {out }}(k)$ in Figure 1(b) becomes steeper. Furthermore, an increase in $\hat{k}$ makes the monopoly profit more sensitive to changes in the average price paid by a consumer's neighbor. Specifically, a marginal increase of the average price paid by a randomly selected neighbor, $\bar{p}$, decreases the monopoly marginal profits by $\sum_{k \in D} P(k) \partial \pi(k) / \partial \bar{p}=\gamma\left[\gamma \sigma_{k}^{2}+2 \hat{k}\right] / 2\left[4-4 \gamma \hat{k}-\gamma^{2} \sigma_{k}^{2}\right]$, which is increasing in $\hat{k}$. Since, as we explained above, it is $\sum_{k \in D} \partial \pi(k) / \partial \bar{p}$ that captures the 


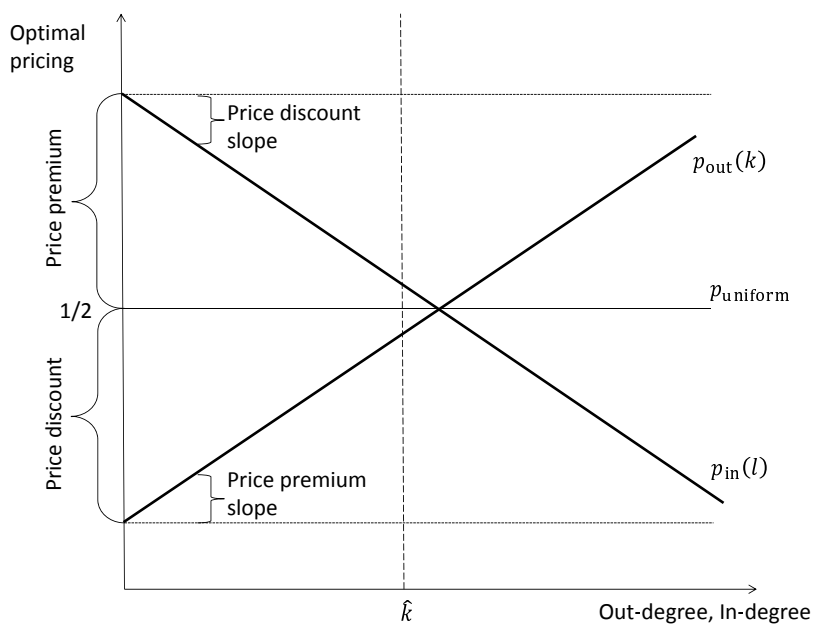

(a) Price discrimination on out-degree and price discrimination on indegrees

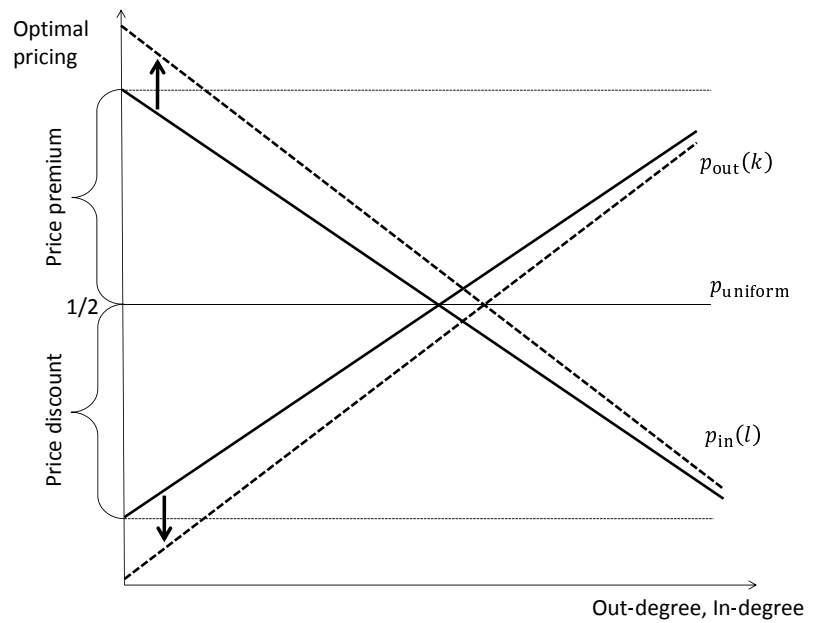

(b) Effect of increasing $\hat{k}$ and/or $\sigma_{k}^{2}$ and $\sigma_{l}^{2}$ on optimal pricing functions

FiguRE 1

Optimal pricing functions.

monopoly's incentives to subsidize consumers for their influence, we have that an increase in $\hat{k}$ increases the price discount offer to consumers - i.e., the intercept of the optimal price function $p_{\text {out }}(k)$ in Figure $1(\mathrm{~b})$ decreases.

We now explain the effect on pricing of a change in the variance of the out-degree 
distribution. When the levels of susceptibility become more dispersed, a consumer who samples consumer $i$ is, on average, more susceptible and, therefore, pays, on average, a higher price. Because now consumer $i$ affects higher paying consumers, she becomes more influential for the monopoly's profits and receives a larger price discount. To see this more formally, recall that the price discount to consumer $i$ for her influence equals $\sum_{k} P(k) \partial \pi(k) / \partial \bar{p}=\sum P(k) p_{\text {out }}(k) k /(1-\gamma \hat{k})$, and since $p_{\text {out }}(k)$ is linear in $k$, the price discount that consumer $i$ receives is proportional to $\sum P(k) k^{2}=\sigma_{k}^{2}+\hat{k}^{2}$, which, in fact, is related to the average out-degree of a randomly chosen consumer who has sampled consumer $i{ }^{17}$ The fact that an increase in $\sigma_{k}^{2}$ leads to a higher price discount to each consumer, also implies that the average price goes down, which boosts peers' consumption and increases consumer surplus. The monopoly, then, extracts this additional consumer surplus from highly susceptible consumers by increasing the price premium.

\subsection{Consumption}

The following proposition shows how the equilibrium consumption depends on consumers' out-degrees and in-degrees.

Proposition 4. The equilibrium consumption levels of a consumer at the optimal price schedule for discrimination on out-degree, $x_{\text {out }}(k, l)$, in-degree, $x_{i n}(k, l)$, and both, $x_{\text {in } / \text { out }}(k, l)$, are the following:

$$
\begin{aligned}
x_{\text {out }}(k) & =\frac{2+\gamma(k-\hat{k})}{4-4 \gamma \hat{k}-\gamma^{2} \sigma_{k}^{2}}, \\
x_{\text {in }}(k, l) & =\frac{2 \hat{k}+\gamma\left(2 \hat{k}+\gamma \sigma_{l}^{2}\right)(k-\hat{k})+\gamma \hat{k}(l-\hat{k})}{\hat{k}\left(4-4 \gamma \hat{k}-\gamma^{2} \sigma_{l}^{2}\right)}, \\
x_{\text {in } / \text { out }}(k, l) & =\frac{1}{2}\left[1+\frac{\left(\gamma \sigma_{l}^{2}+2 \hat{k}\right) k+\left(\gamma \sigma_{k}^{2}+2 \hat{k}\right) l}{\hat{k}^{2}\left(4-4 \hat{k} \gamma-\gamma^{2}\left(\sigma_{k}^{2}+\sigma_{l}^{2}\right)\right)-\gamma^{2} \sigma_{l}^{2} \sigma_{k}^{2}} \hat{k} \gamma\right] .
\end{aligned}
$$

Corollary 2. If the monopoly price discriminates on out-degrees (resp. in-degrees), the equilibrium consumption level of a consumer linearly increases in her out-degree (resp. out-degree and in-degree).

When the monopoly discriminates only on consumers' susceptibility, there are two confounding effects. On the one hand, a highly susceptible consumer pays a high price, and this decreases her consumption; on the other hand, a highly susceptible consumer enjoys more network effects, and this boosts her consumption. The merit of Proposition 4 and Corollary 2 is to show that the optimal prices are set so that more-susceptible consumers consume more. The reason is that the monopoly does not want to forfeit the high price on the large number of units that they consume. For this to hold, it must be the case that the high out-degree consumers consume more than low out-degree ones.

17. Note that the out-degree distribution of an individual at the out end of a randomly chosen link is $\bar{P}(k)=\frac{k}{\hat{k}} P(k)$, and so the expected out-degree of an individual at the end of a randomly chosen link is $\sum_{k} \bar{P}(k) k=\frac{1}{\hat{k}} \sum_{k} P(k) k^{2}=\frac{\sigma_{k}^{2}}{\hat{k}}+\hat{k}$. 
Second, when the monopoly discriminates only on consumers' influence, a consumer's consumption decision depends on her levels of influence and susceptibility, but in a separable way. Given any out-degree, more-influential agents receive a higher price discount, so their consumption is higher than that of less influential agents. At the same time, since the price schedule is independent of the level of susceptibility, more-susceptible agents, by being exposed to higher network effects, consume more.

\section{WELFARE AND THE VALUE OF NETWORK INFORMATION}

We now investigate the effect of targeted pricing strategies on consumers' utilities and monopoly profits.

\subsection{Consumer surplus}

We characterise the effects on consumers' utilities and aggregate consumer surplus of moving from a uniform pricing strategy to a pricing strategy that incorporates information on influence or susceptibility to influence. For the sake of brevity, we do not compare the case of no price discrimination with the case of price discrimination on both in-degrees and out-degrees, but analogous results and intuitions apply to that comparison.

Recall that for a given consumption profile $\left(x_{i}, \mathbf{x}_{-i}\right)$ and price $p_{i}$, the expected utility to consumer $i$ with out-degree $k$ and in-degree $l$ is

$$
U_{i}\left(x_{i}, \mathbf{x}_{-i}, p_{i}, k\right)=\left(1-p_{i}\right) x_{i}-\frac{1}{2} x_{i}^{2}+\gamma x_{i} k A\left(\mathbf{x}_{-i}\right) .
$$

In a Nash equilibrium of the consumption stage, consumer $i$ plays a best reply; that is, $i$ 's consumption $x_{i}^{*}$ satisfies

$$
1-p_{i}-x_{i}^{*}+\gamma k A\left(\mathbf{x}_{-i}^{*}\right)=0 .
$$

We can then rewrite the equilibrium utility of consumer $i$ as follows:

$$
U_{i}^{*}=\frac{1}{2}\left[x_{i}^{*}\right]^{2} .
$$

Therefore, to determine whether a consumer with out-degree $k$ and in-degree $l$ is better off when the seller does not discriminate, as compared to the case where, say, the seller discriminates on out-degree, we need to compare the consumer's equilibrium consumption in the two regimes ${ }^{18}$ Furthermore, aggregating consumers' utilities across different types, we obtain a simple expression for consumer surplus:

$$
C S=\frac{1}{2} \sum_{l \in D} \sum_{k \in D} H(l) P(k)\left[x^{*}(k, l)\right]^{2} .
$$

\section{Proposition 5.}

1. Suppose that we move from no-discrimination to discrimination on out-degrees. There exists a threshold $k^{\prime}>\hat{k}$ so that a consumer is better off if, and only if, her

18. We note that, for a given regime of price discrimination, the equilibrium consumer's utility is increasing in her out-degree; furthermore, when there is discrimination on in-degrees, the equilibrium consumer's utility is also increasing in her in-degree. These follow directly from Corollary 2 and the fact that the equilibrium consumer's utility is increasing in equilibrium consumption. 
out-degree $k \leq k^{\prime}$. The threshold $k^{\prime}$ is increasing in the variance of the out-degree distribution.

2. Suppose that we move from no-discrimination to discrimination on in-degrees. There exists a threshold function $l^{\prime}(k)$, which is decreasing in $k$, so that a consumer with out-degree $k$ and in-degree $l$ is better off if, and only if, her in-degree $l \geq l^{\prime}(k)$.

Price discrimination of any form increases average consumption, which has a positive effect on the utility of each of the consumers through the positive network externalities. At the same time, when the seller discriminates on susceptibility, all consumers receive a price discount relative to the uniform price, and they receive a price premium that is increasing in their level of susceptibility (see Figure 1(a)). Hence, there exists a susceptibility threshold so that only consumers with a level of susceptibility below the threshold benefit from the introduction of such price discrimination. The most interesting part of the proposition, however, is that if the out-degrees are more dispersed, this susceptibility threshold is higher. The basic intuition closely follows the comparative statics illustrated in Figure 1(b). When out-degrees become more dispersed, the monopoly charges a lower price to each consumer. Thus, there is a larger range of consumers, in terms of their out-degrees, who benefit from price discrimination.

When discrimination is on consumers' influence, all consumers receive a constant price premium, and influential consumers receive a price discount. So, among consumers with a certain susceptibility, more-influential consumers benefit from price discrimination on in-degrees. Moreover, the fact that the function $l^{\prime}(k)$ is decreasing in $k$ implies that consumers with a high susceptibility may benefit from discrimination on in-degrees even if they are not as influential as some other consumers with a low susceptibility. Intuitively, by price discriminating, the seller stimulates overall consumption and, therefore, fixing the in-degree of a consumer, highly susceptible consumers enjoy greater network externalities.

In general, the introduction of price discrimination may be beneficial for some consumers but not for others. However, we can prove an unambiguous result for the aggregate consumer surplus.

\section{Proposition 6.}

1. For every $\sigma_{k}^{2}$ and $\gamma$, there exists a $\tilde{k}\left(\sigma_{k}^{2}, \gamma\right)>0$ such that when we move from nodiscrimination to discrimination on out-degrees, consumer surplus increases if, and only if, $\hat{k}>\tilde{k}\left(\sigma_{k}^{2}, \gamma\right)$. Moreover, $\tilde{k}\left(\sigma_{k}^{2}, \gamma\right)$ is decreasing in $\sigma_{k}^{2}$ and $\gamma$.

2. Consumer surplus increases when we move from no-discrimination to discrimination on in-degrees.

The intuition for Proposition 6 requires noting a few simple observations. First, with linear pricing, a consumer's equilibrium utility is increasing and convex in equilibrium consumption 19 This implies that increasing the average and/or the dispersion of equilibrium consumption across individuals increases consumer surplus. The second observation is that price discrimination, regardless of whether the discrimination is on consumers' influence or consumers' susceptibility, increases average consumption. On the other hand, the effect of price discrimination on the dispersion of equilibrium consumption

19. This observation applies to any model with linear pricing and concave utility function, and is akin to the traditional consumer surplus triangle under the demand curve. In our quadratic utility model, this is captured by $U_{i}=\frac{1}{2}\left[x_{i}^{*}\right]^{2}$. 


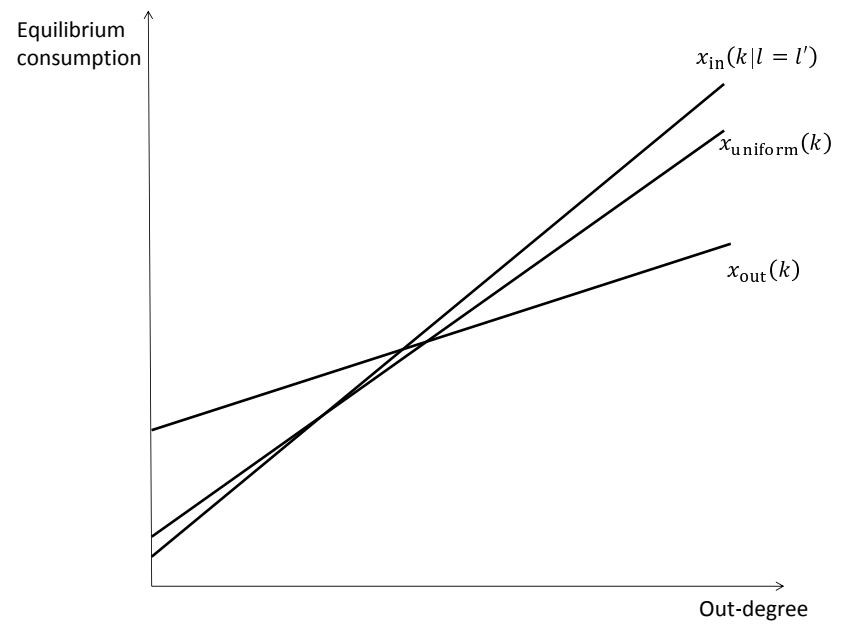

FiguRE 2

Equilibrium consumption: uniform price vs price discrimination.

depends on whether the monopoly discriminates on in- or out-degrees. In particular, price discrimination based on consumers' influence increases the dispersion of equilibrium consumption across consumers, whereas discrimination on consumers' susceptibility has the opposite effect. Figure 2 contrasts equilibrium consumption as a function of outdegree for the case of no price discrimination with the case of price discrimination on out-degrees and for the case of price discrimination on in-degrees.

Discrimination based on susceptibility is akin to more traditional forms of price discrimination absent of network effects - i.e., charging higher prices to consumers whose demand schedule is higher. Thus, its implication is to dampen the quantity consumed by high-demand consumers, and, therefore, it decreases the dispersion in equilibrium consumption. However, a consumer's level of influence does not, ceteris paribus, affect her demand, and, therefore, discrimination based on influence does not dampen the consumption of highly susceptible consumers. Moreover, price discrimination on influence leads to an increase in the consumption of influential consumers and, thus, to an increase in network effects. While this increase affects all individuals, it affects highly susceptible consumers more, leading to an increase in the dispersion of consumption across consumers.

Propositions 5 and 6 cover situations in which a consumer obtains positive externality from her peers' consumption. In some other situations, such as smoking, peers' consumption has negative externalities. A way to incorporate this is to modify the preferences of consumer $i$ as follows:

$$
u_{i}\left(x_{i}, \mathbf{x}_{-i}, p_{i}\right)=x_{i}-\frac{1}{2} x_{i}^{2}+\gamma x_{i} \sum_{j \in N_{i}} x_{j}-\lambda \sum_{j \in N_{i}} x_{j}-p_{i} x_{i},
$$

where $\lambda \geq 0$ captures the extent of negative externalities. This modification does not 
affect consumers' marginal utilities (i.e., the nature of network effects); therefore the characterization of consumption behavior, Proposition 1, as well as all the results in Section 3 and in the remainder of the paper still hold. Hence, when $\lambda>0$, we can rewrite the equilibrium expected utility of a consumer with out-degree $k$ as follows $U_{i}^{*}=\frac{\left[x_{i}^{*}\right]^{2}}{2}-\lambda k A\left(\mathbf{x}_{-i}^{*}\right)$.

It is then clear that, since price discrimination on in-degree and/or out-degree boosts average consumption, the presence of negative externalities adds a negative effect on the level of consumers' utilities, and this effect is stronger for more-susceptible consumers. In this case, to draw a final conclusion on how targeted pricing strategies affect consumers' utilities and aggregate consumer surplus, this new negative effect needs to be weighted with the effects that we have emphasised for the case of $\lambda=0$.

\subsection{Profits and the value of network information}

We now describe how the monopoly profits depend on the characteristics of the network and the monopoly's knowledge about the network.20

Proposition 7. The optimal profits for discrimination on out-degree, in-degree, and both, are the following:

$$
\begin{aligned}
\Pi_{\text {out }} & =\frac{1}{4-4 \gamma \hat{k}-\gamma^{2} \sigma_{k}^{2}} \\
\Pi_{\text {in }} & =\frac{1}{4-4 \gamma \hat{k}-\gamma^{2} \sigma_{l}^{2}} \\
\Pi_{\text {in } / \text { out }} & =\frac{1}{4} \frac{4 \hat{k}^{2}-\gamma^{2} \sigma_{k}^{2} \sigma_{l}^{2}}{4 \hat{k}^{2}(1-\gamma \hat{k})-\gamma^{2} \hat{k}^{2}\left(\sigma_{k}^{2}+\sigma_{l}^{2}\right)-\gamma^{2} \sigma_{k}^{2} \sigma_{l}^{2}}
\end{aligned}
$$

Corollary 3. For all price discrimination schemes, the profit of the monopoly is increasing and convex in the average level of network effects. Furthermore, it is increasing and convex in the variance of the out-degree distribution (resp. in-degree distribution) if the monopoly price discriminates on out-degrees (resp. in-degrees).

Since consumers' demand and optimal pricing strategy are both linear in consumers' type, the average level of network effects and the variance of the out-degree and the indegree distribution are sufficient to compute the profits that a monopoly would obtain by price discriminating. This gives a precise guide to practitioners for prioritizing their data collection/purchase decisions. It also allows companies, such as Klout, Facebook, MySpace, Google, and the like, which own data on social interactions and influence, to provide firms with sufficient statistics to convey the value of the data without revealing the data itself —also known as zero-knowledge information transmission in the cryptography literature. ${ }^{21}$

Corollary 3 shows how changes in the pattern of network effects alter the expected profit. We now develop the intuitions for the case of price discrimination on out-degrees.

20. We also note that the additional profit for the monopoly from introducing any type of price discrimination is greater than any loss to the consumer surplus. That is, aggregate welfare increases when we move from no discrimination to discrimination on in-degrees or out-degrees.

21. For a classic example, see Feige et al. (1988) 
Note, first, that for a given price schedule, an increase in $\hat{k}$ is synonymous with an increase in network effects, and this triggers higher demand of each consumer and higher monopoly profits, ceteris paribus. Hence, at the new optimal price schedule, the monopoly's profit must also increase. Next, recall that consumers' demand and the price they are charged increase linearly in their susceptibility. So, the monopoly profit from a consumer is increasing and convex in her level of susceptibility. An increase in the variance of the out-degree distribution, therefore, increases the expected profit of the monopoly, ceteris paribus. Hence, at the new optimal price schedule, the profit of the monopoly must also increase.

We now investigate the characteristics of the network effects across consumers that makes the monopoly willing to acquire information on consumers' influence. The value to the monopoly of learning the out-degree (resp. in-degree) relative to no-information is defined as the difference in the expected profit when the monopoly discriminates on out-degrees (resp. in-degree) and the expected profit under uniform price-i.e., $\Pi_{\text {out }}-\Pi$ (resp. $\left.\Pi_{i n}-\Pi\right)$. We then say that the monopoly prefers to learn the out-degree rather than the in-degree when the value of learning the out-degree is higher than the value of learning the in-degree. Corollary 4 answers the following questions: How does the value of learning in-degrees and out-degrees depend on the pattern of network effects? If the monopoly can acquire only information on either the influence of consumers or their susceptibility, which information should the monopoly acquire?

Corollary 4. The value to the monopoly from learning the out-degrees (resp. in-degrees) of all consumers relative to having no information on individual consumers' out- and in-degrees is increasing in the average level of network effects and in the variance of the out-degree (resp. in-degree) distribution. Moreover, the monopoly prefers to learn individual consumers' out-degrees rather than their in-degrees if and only if the variance of the out-degree distribution is larger than the variance of the in-degree distribution.

Recall that the monopoly's profit under uniform price is independent of the variance of the degree distributions, whereas its profit is increasing in the variance of the degree distributions under price discrimination. Hence, the value of information on indegrees or out-degrees is higher when their respective distributions are more dispersed ${ }^{22}$ Furthermore, whether the monopoly prefers to learn consumers' influence or their susceptibility depends solely on the variances of the corresponding distributions: the monopoly prefers to learn the degrees that have higher variance, as a high spread allows for more-profitable price discrimination. The fact that the value of information on consumers' influence or consumers' susceptibility is higher for products with a higher average level of network effects is less immediate because in the case of uniform price, the monopoly profits are also increasing in $\hat{k}$. Corollary 4 essentially shows that by price discriminating, the monopoly can leverage the increase in the average network externalities more effectively.

One question that is not answered by Corollary 4 is: When will a monopoly invest in learning both the influence and the susceptibility of consumers, as opposed to investing only in information on the more valuable of the two? In other words, we would like to know whether the value of information is separable, or whether the data on consumers' out-

22. It is also the case that the value to the monopoly of learning the in-degrees and out-degrees relative to no-information is increasing in the variance of the in-degree distribution and in the variance of the out-degree distribution. 
and in-degrees complement or substitute for each other in terms of generating monopoly profit.

Definition 1. We say that the value to the monopoly from learning the out-degrees and in-degrees for all consumers exhibits complementarity if:

$$
\left.\Pi_{\text {in } / \text { out }}-\Pi_{\text {out }} \geq \Pi_{\text {in }}-\Pi \quad \text { (or equivalently } \Pi_{\text {in } / \text { out }}-\Pi_{\text {in }} \geq \Pi_{\text {out }}-\Pi\right) .
$$

If the inequalities are reversed we say that the value for the monopoly from learning the out-degrees and in-degrees of all consumers exhibits substitutability.

Corollary 5. The value to the monopoly from learning the out-degrees and in-degrees of all consumers exhibits complementarity.

Corollary 5 implies that the monopoly values having data on individual consumers' influence more if the monopoly already has data on individual consumers' susceptibility, and vice-versa. An immediate implication is that if the variances of the in- and outdegree distributions are equal (which implies that the values of learning the in-degree or out-degree are equal), and if the cost of acquiring each of the different data is the same, then a monopoly will either invest in collecting data on both consumers' influence and susceptibility or on neither.

So far, we have conducted the analysis under the assumption that the monopoly knows the out-degree and/or in-degree of all consumers. In reality, the monopoly may have information on only a subset of the population. What is the marginal value of learning some consumers' in-degrees or out-degrees, without being able to implement full price discrimination on everyone's types?

We now briefly show how to accommodate these considerations in our model and the insights we can obtain. We focus on the case of price discrimination on consumers' susceptibility, and we assume that the monopoly has information about the out-degree of a fraction of consumers $y \in[0,1]$, but it has no information about the influence and susceptibility of other consumers 23 We also assume that the fraction $y$ of consumers is a representative sample, in the sense that a fraction $P(k)$ of targeted consumers have out-degree $k$, for all $k \in D$. The monopoly sets a price $p_{\text {out }}(k)$ to targeted consumers with out-degree $k \in D$, and sets a constant price $p$ to the other $1-y$ consumers.

In equilibrium, the demands of a targeted consumer and of a non-targeted consumer with out-degree $k$ are

$$
x^{T}(k)=1+\gamma k A(\mathbf{x})-p_{\text {out }}(k) \text { and } x(k)=1+\gamma k A(\mathbf{x})-p,
$$

respectively. The problem of the monopoly is, then, to choose $\left\{p_{\text {out }}(k)\right\}_{k \in D}$ and $p$ to maximize

$$
\Pi=\sum_{k \in D} P(k)\left[y x^{T}(k) p_{\text {out }}(k)+(1-y) x(k) p\right],
$$

where $x^{T}(k)$ and $x(k)$ are given above. Using the same logic we have developed in the analysis for the case of $y=1$, we can solve for the optimal pricing strategy, and we obtain

23. The results we highlight here translate easily to the case of price discrimination on in-degrees and price discrimination on in-degrees and out-degrees. For example, in the case of price discrimination on in-degrees we get an analogous result from the one illustrated below: non targeted consumers receive a price $p=1 / 2+y \gamma^{2} \sigma_{l}^{2} / 2\left[4-4 \gamma \hat{k}-y \gamma^{2} \sigma_{l}^{2}\right]$ and targeted consumers with in-degree $l$ receive a price $p_{\text {in }}(l)=p-\gamma(l-\hat{k}) /\left[4-4 \gamma \hat{l}-y \gamma^{2} \sigma_{l}^{2}\right]$ 
that the price charged to a non targeted consumer is

$$
p=\frac{1}{2}-\frac{1}{2} \frac{y \gamma^{2} \sigma_{k}^{2}}{4-4 \gamma \hat{k}-y \gamma^{2} \sigma_{k}^{2}} .
$$

and the price charged to a targeted consumer with out-degree $k$ is

$$
p_{\text {out }}(k)=p+\frac{\gamma(k-\hat{k})}{4-4 \gamma \hat{k}-y \gamma^{2} \sigma_{k}^{2}} .
$$

The price charged to non-targeted consumers is the average price charged to the targeted consumers. Relative to the case of pure uniform price $(y=0)$, the possibility for the monopoly to price discriminate on consumers' susceptibility creates a spillover to the price received by non-targeted consumers. The monopoly gives a price discount to nontargeted consumers because they influence the consumption of targeted consumers. We can go further and compute equilibrium profit and obtain that

$$
\Pi_{\text {out }}(y)=\frac{1}{4(1-\gamma \hat{k})-\gamma^{2} \sigma_{k}^{2} y} .
$$

The monopoly's profit is increasing and convex in the fraction $y$ of targeted consumers. Furthermore, the marginal increase in the profit due to a marginal increase in $y$ is increasing in the dispersion of the out-degree distribution-i.e., the cross partial derivative of the $\Pi_{\text {out }}$ with respect to $y$ and $\sigma_{k}^{2}$ is positive. This result shows that the insights on the value of information that we derived by comparing the monopoly profits under no price-discrimination, $y=0$, and the monopoly profits under price discrimination, $y=1$, are robust to the case in which the monopoly can price discriminate only on a subset $y$ of consumers.

\section{CORRELATION BETWEEN INFLUENCE AND SUSCEPTIBILITY}

Building on the generality of our framework, we study how monopoly profits and the value of information are affected by the correlation in the population between consumers' influence and consumers' susceptibility-henceforth, degree correlation. Negative correlation between influence and susceptibility is typical of many goods, such as [1] goods that have an element of expertise, such as prescription choices by physicians, in which case few experts act as opinion leaders; [2] professional goods adopted in superior-inferior relationships, such as communication devices in hierarchical organizations; and [3] fashionable goods, such as designer clothing brands, which are often endorsed by celebrities ${ }^{24}$ Positive correlation is, instead, typical of social goods, in which social interaction is more symmetric, such as Facebook.com usage time and communication products, including communication applications on smartphones. Is the expected monopoly's profit higher or lower when the big influencers in a market are not influenced much by others? How does the willingness of the monopoly to invest in

24. Tucker (2008), in a study of the adoption of video-conferencing technology in a large multinational organization, shows that adoption and usage of the technology by employees is influenced by the usage of the technology by their superiors, but less so by their subordinates. Nair et al. (2010) report asymmetric influence between prominent physicians and non-specialist physicians in the context of prescription and adoption of new drugs. Aral and Walker (2012), in a study of adoption of an application on Facebook.com, also show the importance of distinguishing between influential and susceptible consumers. Goldenberg et al. (2009), in a study of adoption of pictures and video-clips in a social networking website, show that influence and susceptibility to influence are both important dimensions on which to understand diffusion and to identify hubs. 
gathering information depend on whether the more susceptible consumers also influence others?

Let $P(k \mid l)$ be the probability that a node with in-degree $l$ has out-degree $k$, and let $H(l \mid k)$ be the probability that a node with out-degree $k$ has in-degree $l$. By definition, the following consistency conditions apply: for all $l$ and $k, \sum_{l} P(k \mid l) H(l)=P(k)$ and $\sum_{k} H(l \mid k) P(k)=H(l)$. For simplicity, we assume in this section that the variances of the in- and out-degree distributions are the same-i.e., $\sigma_{k}^{2}=\sigma_{l}^{2}=\sigma^{2}$. Therefore, the correlation coefficient can be written as

$$
\rho=\frac{\operatorname{Cov}(k, l)}{\sigma^{2}}=\frac{E[(k-\hat{k})(l-\hat{k})]}{\sigma^{2}}=\frac{E[k l]-\hat{k}^{2}}{\sigma^{2}} .
$$

The case in which there is no correlation obtains when $\rho=0$. Before proceeding with the analysis, we briefly describe the results for perfect positive correlation. This conveys some basic intuitions that are useful to understanding the general analysis.

Example: Perfect Positive Correlation. Perfect positive correlation occurs when $\rho=1$. In this case, if the monopoly knows consumers' susceptibility, it also knows their influence, and vice-versa. Hence, the optimal price function is the same regardless of whether the monopoly has information on consumers' in- or out-degrees (or both). We now show that, when $\rho=1$, the monopoly always prices as if it has no information about the degrees of consumers: the optimal price equals $1 / 2$ for all consumers.

Consider that the monopoly price discriminates on out-degrees. As in the benchmark case, the monopoly applies a price premium, which is increasing in the out-degree, and a price discount that reflects the influence that the consumer has on her peers. When susceptibility and influence are independent, the price discount captures the average consumer's influence on her peers. Under perfect positive correlation, the monopoly knows that the consumer with out-degree $k$ influences exactly $k$ other consumers. So, it applies a price discount that is increasing in $k$. The linearity in the model that results from consumers' quadratic utilities, implies that the price premium and the price discount cancel each other out.

Nevertheless, the profit of the monopoly under perfect correlation is higher than under independence. To see this, start from the optimal price function when consumers' susceptibility and influence are independent. Recall that consumption is increasing in consumers' susceptibility and it is independent of consumers' level of influence. Now consider a change from independence to perfect correlation. Keeping everything else constant, each consumer expects a higher average peer's consumption because, now, the most susceptible consumers are also the most influential. This increase in network effects boosts consumers' demand and, therefore, increases the monopoly's profit. ॥

We now turn to the analysis for arbitrary levels of correlation. When the monopoly price discriminates on in-degree (resp. out-degree), it turns out that in determining the optimal price schedule, the monopoly will take into account the average out-degree (resp. in-degree) of a consumer conditional on the consumer's in-degree (resp. out-degree)-i.e., $\hat{k}(l)=\sum_{k} P(k \mid l) k$, and the variance of this random variable, $\sigma_{\hat{k}(l)}^{2}=\sum_{l} H(l)[\hat{k}-\hat{k}(l)]^{2}$ (resp. $\hat{l}(k)$ and $\left.\sigma_{\hat{l}(k)}^{2}\right)$. For general levels of correlation, there is no specific relationship between $\hat{k}(l)$ and $\sigma_{\hat{k}(l)}^{2}$ and the first and second moments of the distribution functions $P$ and $H$. We can derive the optimal price function and the equilibrium consumption at the optimal price for arbitrary correlations and for different regimes of price discrimination (see online Appendix I). However, to understand how a change in the level of correlation affects profits, we need to impose some structure on the relationship between these 
conditional random variables and the moments of the distributions of in-degree and out-degree. Assumption 2 provides the tractability that allows us to prove Proposition 8 and Proposition 9

Assumption 2. The correlation between the in-degree and the out-degree is such that their conditional expectations are linear-i.e., $\hat{k}(l)=E[k \mid l]$ is linear in $l$ and $\hat{l}(k)=E[l \mid k]$ is linear in $k$.

Assumption 2 allows for a large family of joint distributions, including the family of the so-called bounded elliptical distributions, which are used extensively in the finance literature, see, e.g., Owen and Rabinovitch (1983).25 Assumption 2 does not restrict correlation from being negative or positive, and $\rho$ can take any value in the range $[-1,1]$ as before. However, the assumption implies that $\hat{k}(l)=\hat{k}+\rho(l-\hat{k}), \hat{l}(k)=\hat{k}+\rho(k-\hat{k})$, and $\sigma_{\hat{l}(k)}^{2}=\sigma_{\hat{k}(l)}^{2}=\rho^{2} \sigma^{2}{ }^{26}$

In what follows, we focus on describing results on how correlation affects both optimal profit and the value of network information for the monopoly.

Proposition 8. Suppose that Assumption 2 holds. Regardless of whether the monopoly discriminates based on in-degree, out-degree, both, or none, the monopoly's profit increases in $\rho$.

Consistent with the intuition for the case of perfect correlation, an increase in the correlation between susceptibility and influence means that the high-demand consumers are now more likely to be also influential. This has positive feedback for the consumption decisions of other consumers and, so, the monopoly earns a higher profit.

Proposition 9. Suppose that Assumption 2 holds. The value to the monopoly of learning either the out-degrees or the in-degrees relative to no-information is decreasing in $\rho$. Furthermore, there exists a $\rho^{*} \in(-1,0)$ such that the value to the monopoly from learning the out-degrees and in-degrees of all consumers exhibits substitutability for every $\rho<\rho^{*}$, and complementarity otherwise.

Aral and Walker (2012) study the adoption of an application on Facebook.com and show that, in their data, influence and susceptibility to influence are negatively correlated. Proposition 9 tells us that negative correlation increases the price that a firm selling

25. We refer to Kazaz et al. (2005) and Van Mieghem (1995) for a treatment of elliptical distributions.

26. A weaker, but qualitatively similar, statement of Proposition 8 and Proposition 9 can be proved under a much weaker assumption than Assumption 2 Consider, indeed, the weak assumption that $\hat{k}(l)$ and $\hat{l}(k)$ are both continuous functions in $\rho \in[-1,1]$. This includes the environments covered by Assumption 2 but also many others, with both monotone and non-monotone conditional expectation functions. Under this weaker assumption, we can prove the following counterpart of Proposition 8 Regardless of whether the monopoly price discriminates based on in-degree, out-degree, both, or none, there exists $\varepsilon>0$ such that the monopoly's profit is higher when $\rho \in[1-\varepsilon, 1]$ than when $\rho \in[-\varepsilon, \varepsilon]$, and it is higher when $\rho \in[-\varepsilon, \varepsilon]$ than when $\rho \in[-1,-1+\varepsilon]$. Under this weaker assumption, we can also prove the following counterpart of Proposition 9 There exists $\varepsilon>0$ such that the value to the monopoly for learning either the out-degrees or the in-degrees relative to no-information is higher when $\rho \in[-1,-1+\varepsilon]$ than when $\rho \in[-\varepsilon, \varepsilon]$, and higher when $\rho \in[-\varepsilon, \varepsilon]$ than when $\rho \in[1-\varepsilon, 1]$. Furthermore, when $\rho \in[-1,-1+\varepsilon]$, the value to the monopoly from learning the out-degrees and indegrees of all consumers exhibits substitutability, whereas when $\rho \in[-\varepsilon, \varepsilon]$ or $\rho \in[1-\varepsilon, 1]$, it exhibits complementarity. 
such an application will be willing to pay Facebook.com for its network data. Moreover, the negative correlation suggests that information on influence and on susceptibility are substitutes (or weak complements). In turn, Proposition 9 prescribes that a firm selling this type of application on Facebook should invest in learning only one of the distributions (assuming equal costs of acquiring each distribution).

The intuition behind Proposition 9 is also related to the discussion of the optimal price under perfect positive correlation. Optimal price discrimination entails a price premium that is increasing in the consumer's susceptibility, and a price discount which is increasing in consumer's influence. Increased correlation between susceptibility and influence implies, then, that the price premium effect and the price discount effect tend to cancel each other out. This has two implications.

First, the value of network information is decreasing in the correlation between susceptibility and influence. Second, when highly influential consumers are also very susceptible, information only on a consumer's susceptibility (resp. influence) is not particularly useful for price discrimination. Charging a high price premium to consumers who are highly susceptible can be harmful because these consumers are likely to be very influential. At the same time, information on both in-degrees and out-degrees allows the monopoly to detect consumers who are highly susceptible but not very influential, and the monopoly can effectively price discriminate to these consumers. So, under high positive correlation, even though the value of either kind of information on degrees is low, the information on consumers' influence and consumers' susceptibility are complements. ${ }^{27}$

\section{CONCLUSION}

Online commerce generates data on local network effects that were not available earlier. This shift in the information that firms can use when designing their marketing strategies motivates an array of important economic questions. In this paper, we have developed a framework for studying optimal pricing in the presence of network effects. The analysis provides clear-cut predictions on how a monopoly should incorporate detailed information on network effects in its pricing strategy, as well as the implications for consumer surplus and profits.

Our analysis has focused on the case of a monopoly firm. A particularly interesting direction in which our model should be extended is to consider the effects of multiple firms simultaneously using information on consumers' network effects. For example, one would like to understand how competition across firms affects the way that firms incorporate network effects in their pricing strategies. Equally interesting is the case in which firms sell complementary network goods and have access to detailed information on network effects.

Another interesting extension is to endogenize influence. We see hints of that in websites such as Klout.com, where individuals are encouraged to develop their influence

27. This result is particularly surprising. To see why, consider a decision maker who must choose between actions whose outcome depends on the realizations of two random variables. The decision maker knows the underlying distribution of both random variables, but needs to pay to learn their realizations before making a decision (or make a decision without such information). Then, both positive and negative correlations between the random variables make learning the realization of a second random variable less valuable than it would have been to learn it without having the first piece of information. In particular, if correlation is almost perfect (either positive or negative), then for any reasonable payoff function, the information on the two random variables would exhibit substitutability. Thus, it is the subtle interplay between network effects and the use of the information that the monopoly has about these externalities, that generates the complementarity result. 
in order to receive discounts via 'Klout Perks.' We note that, in our model, an individual always wants to be both more influential and more susceptible to influence. However, the marginal benefit of an additional in- and out-link varies according to the information that the monopoly uses in its pricing. In a world in which establishing and maintaining links is costly, patterns of influence will be affected by the information available to the monopoly. A somewhat easier related question is how much information on their influence and susceptibility consumers will choose to reveal to the monopoly. This can, at least in principle, be modeled using second-degree price discrimination.

Finally, this paper focuses on the important case of positive network effects-i.e., consumption decisions are strategic complements. Nevertheless, our analysis and solution apply also to the case of strategic substitutes, as long as the negative influence is not too strong.

\section{APPENDIX A.}

We first state and prove equilibrium existence and uniqueness in the consumption stage in a model where we allow general correlations between in-degrees and out-degrees, i.e., Proposition A10 We then provide a sufficient condition for the monopoly problem to have an interior solution, i.e., Proposition A11. We note that the arguments developed in the proofs of these two propositions do not rely on whether out-degrees and in-degrees are correlated. We define

$$
\bar{k} \equiv \sum_{l} \bar{H}(l) \sum_{k} P(k \mid l) k=\frac{1}{\hat{k}} \sum_{l} \sum_{k} H(l) P(k \mid l) k l=\frac{E[k l]}{\hat{k}},
$$

We also define the expected price paid by a randomly selected neighbours of a consumer as

$$
\bar{p} \equiv \sum_{l} \bar{H}(l) \sum_{k} P(k \mid l) p(k, l)
$$

In the case where in-degrees and out-degrees are independent, we have that $\bar{k}=\hat{k}$ and $\bar{p}=$ $\sum_{l} \sum_{k} \bar{H}(l) P(k) p(k, l)$.

Proposition A10. Let $\mathbf{p}$ be any price schedule set by the monopoly in the first stage of the game and specifying for each consumer $i$ a price per unit $p_{i} \in \mathbb{R}$.

1 If $\gamma k^{\max }<1$, then, in the consumption stage, there exists a unique Bayes-Nash equilibrium $\mathbf{x}^{*}$.

2 Assume that $\gamma k^{\max }<1$ and assume that according to $\mathbf{p}$ any two consumers with identical inand out-degrees are priced the same. Then, in the unique equilibrium of the consumption stage any two consumers with identical in- and out-degrees make the same consumption decision. In particular, let $p(k, l)$ and $x(k, l)$ be the price and the demand of a consumer with out-degree $k$ and in-degree $l$. Then, in the unique equilibrium of the consumption stage

$$
x(k, l)=1-p(k, l)+\frac{\gamma(1-\bar{p})}{1-\gamma \bar{k}} k .
$$

\section{Proof of Proposition A10}

Existence: Write the gross utility of consumer $i$ with out-degree $k_{i}$ as $u_{i}\left(k, x_{i}, \mathbf{x}_{-i}\right)=x_{i}-\frac{1}{2} x_{i}^{2}+$ $\gamma k x_{i} A\left(\mathbf{x}_{-i}\right)$. Then, adapting Proposition 1 from Glaeser and Scheinkman (2002) to our setup we get that there exists at least one consumption equilibrium if $\forall_{k \in \mathbb{Z}^{+}, p \in \mathbb{R}} \exists_{\bar{x} \geq 0} \forall_{x \leq \bar{x}} \frac{\partial u}{\partial x_{i}}\left(k, \bar{x},(x)_{j \neq i}\right)-p \leq 0$. To see that this condition holds if $\gamma k^{\max }<1$ it is sufficient to note that $\forall_{k \in \mathbb{Z}^{+}, p \in \mathbb{R}} \exists_{\bar{x} \geq 0} \forall_{x \leq \bar{x}} \frac{\partial u}{\partial x_{i}}\left(k, \bar{x},(x)_{j \neq i}\right)$ $p \leq \frac{\partial u}{\partial x_{i}}\left(k^{\max }, \bar{x},(\bar{x})_{j \neq i}\right)-p=1-\bar{x}+\gamma k \bar{x}-p$ and that $\forall_{p \in \mathbb{R}} \exists_{\bar{x} \geq 0} 1-\bar{x}+\gamma k \bar{x} \leq 0$ if and only if $\gamma k^{\max } \leq 1$.

Uniqueness: Proposition 3 in Glaeser and Scheinkman 2002) implies that there exists at most one consumption equilibrium if $\forall_{i}\left|\frac{\partial^{2} u_{i}}{\partial x_{i} \partial A\left(\mathbf{x}_{-i}\right)} / \frac{\partial^{2} u_{i}}{\left(\partial x_{i}\right)^{2}}\right|<1$ or $\gamma k^{\max }<1$. 
Finally, in an interior equilibrium, for each type $(k, l)$

$$
x(k, l)=1-p(k, l)+\gamma k A\left(\mathbf{x}_{-i}\right),
$$

and therefore

$$
A\left(\mathbf{x}_{-i}\right)=\sum_{l} \bar{H}(l) \sum_{k} P(k \mid l) x(k, l)=1-\bar{p}+\gamma \bar{k} A\left(\mathbf{x}_{-i}\right) \Longleftrightarrow A\left(\mathbf{x}_{-i}\right)=\frac{1-\bar{p}}{1-\gamma \bar{k}} .
$$

Plugging back to the expression for $x(k, l)$ we obtain an explicit expression for the equilibrium consumption of type $(k, l)$. $\|$

Proposition A11. Suppose that $\gamma k^{\max }<\frac{1}{2}$. Then, for any of the price discrimination schemes considered, there exists a unique finite price schedule $\mathbf{p}$ that solves the monopoly's profit maximization problem. This result holds regardless of the correlation between in-degree and out-degree.

Proof of Proposition A11. The proof is available in online Appendix IV.

We now prove all the results in the paper for the case where in-degrees and out-degrees are independent.

Proof of Proposition 1. The Proposition is an immediate implication of Proposition A10

Proof of Proposition 2, Suppose the monopolist only knows the in-degree and out-degree distribution, i.e., $p(k, l)=p$ for all $(k, l)$. Then $\bar{p}=p$, and

$$
x(k, l)=x(k)=1-p+\frac{\gamma(1-p)}{1-\gamma \hat{k}} k .
$$

The monopoly maximizes

$$
\Pi_{\text {uniform }}=\sum_{k} P(k) p x(k)=p(1-p)\left[1+\frac{\gamma \hat{k}}{1-\gamma \hat{k}}\right] .
$$

Hence, $p^{*}=1 / 2$. Simple algebra implies that $x^{*}(k)=\frac{1}{2}\left[1+\frac{\gamma}{1-\gamma \hat{k}} k\right]$ and $\Pi_{\text {uniform }}^{*}=\frac{1}{4}\left[1+\frac{\gamma \hat{k}}{1-\gamma \hat{k}}\right]$. This analysis, in combination with Proposition A11. completes the proof. ॥

Proof of Proposition 3 . Consider first the case of price discrimination on out-degrees. Let $p(k, l)=p(k)$ for all $l \in D$, and so

$$
x(k, l)=x(k)=1-p(k)+\frac{\gamma(1-\bar{p})}{1-\gamma \hat{k}} k,
$$

where $\bar{p}=\sum_{l} \sum_{k} \bar{H}(l) P(k) p(k)$. The monopoly chooses $p(k)$ for all $k \in D$, to maximize $\Pi_{\text {out }}=$ $\sum_{k} P(k) p(k) x(k)$. Taking the derivative with respect to $p(s)$, for some $s \in D$, we have

$$
\frac{\partial \Pi_{\text {out }}}{\partial p(s)}=0 \Longleftrightarrow 1-2 p^{*}(s)+\frac{\gamma}{1-\gamma \hat{k}}\left[\left(1-\bar{p}^{*}\right) s-\sum_{k} P(k) p^{*}(k) k\right]=0 .
$$

Rearranging and denoting $\phi=\sum_{k} P(k) p^{*}(k) k$, we have

$$
p^{*}(s)=\frac{1}{2}\left[1+\frac{\gamma}{1-\gamma \hat{k}}\left[\left(1-\bar{p}^{*}\right) s-\phi\right]\right] .
$$

Combining the definition of $\bar{p}$ and expression $\mathrm{A6}$, we obtain

$$
\bar{p}^{*}=\frac{1}{2}\left[1+\frac{\gamma}{1-\gamma \hat{k}}\left[\left(1-\bar{p}^{*}\right) \hat{k}-\phi\right]\right] .
$$

Combining $\phi=\sum_{k} P(k) p^{*}(k) k$ and expression A6 we derive:

$$
\phi=\frac{1}{2}\left[\hat{k}+\frac{\gamma}{1-\gamma \hat{k}}\left(\left(1-\bar{p}^{*}\right)\left(\sigma_{k}^{2}+\hat{k}^{2}\right)-\phi \hat{k}\right)\right] .
$$


Solving these two last equations for $\bar{p}^{*}$ and $\phi$, and then plugging these expressions back in expression A6 for $p^{*}(s)$, we obtain that the optimal price charged to a consumer with out-degree $k \in D$ is

$$
p_{\text {out }}(k)=\frac{1}{2}+\frac{\gamma k}{4-4 \gamma \hat{k}-\gamma^{2} \sigma_{k}^{2}}-\frac{\gamma}{2} \frac{\gamma \sigma_{k}^{2}+2 \hat{k}}{4-4 \gamma \hat{k}-\gamma^{2} \sigma_{k}^{2}} .
$$

Second, consider the case of price discrimination on in-degrees. Let $p(k, l)=p(l)$ for all $k, l \in D$, and note that equilibrium consumption is

$$
x(k, l)=1-p(l)+\frac{\gamma(1-\bar{p})}{1-\gamma \bar{k}} k,
$$

where, in this case, $\bar{p}=\frac{1}{\hat{k}} \sum_{l} H(l) l p(l)$. The profits are $\Pi_{i n}=\sum_{l} \sum_{k} H(l) P(k) p(l) x(k, l)$. Taking the derivative of $\Pi_{i n}$ with respect to the price charged to consumers with in-degree $s \in D$ we obtain

$$
\frac{\partial \Pi_{i n}}{\partial p(s)}=0 \Longleftrightarrow p^{*}(s)=\frac{1}{2}\left[1+\frac{\gamma}{1-\gamma \hat{k}}\left(\left(1-\bar{p}^{*}\right) \hat{k}-s \phi\right)\right]
$$

where $\phi=\sum_{l} H(l) p^{*}(l)$. Using $p^{*}(s)$ and the definition of $\bar{p}$ we obtain that

$$
\bar{p}^{*}=\frac{1}{2}\left[1+\frac{\gamma}{1-\gamma \hat{k}}\left(\left(1-\bar{p}^{*}\right) \hat{k}-\phi \frac{\sigma_{l}^{2}+\hat{k}^{2}}{\hat{k}}\right)\right] .
$$

Similarly, using $p^{*}(s)$ and the definition of $\phi$ we obtain

$$
\phi=\frac{1}{2}\left[1+\frac{\gamma \hat{k}}{1-\gamma \hat{k}}\left(1-\bar{p}^{*}-\phi\right)\right]
$$

Solving the two equations for $\phi$ and for $\bar{p}^{*}$, and plugging the obtained expressions on the expression for $p^{*}(s)$, we obtain that the price charged to a consumer with in-degree $l \in D$ is:

$$
p_{i n}(l)=\frac{1}{2}+\frac{\gamma}{2} \frac{\gamma \sigma_{l}^{2}+2 \hat{k}}{4-4 \gamma \hat{k}-\gamma^{2} \sigma_{l}^{2}}-\frac{\gamma l}{4-4 \gamma \hat{k}-\gamma^{2} \sigma_{l}^{2}} .
$$

Finally, we turn to the case of price discrimination on in-degrees and out-degrees. The derivation follows the same steps derived in the previous two cases. Equilibrium consumption is:

$$
x(k, l)=1-p(k, l)+\frac{\gamma(1-\bar{p})}{1-\gamma \bar{k}} k,
$$

where, in this case, $\bar{p}=\sum_{l} \sum_{k} \bar{H}(l) P(k) p(k, l)$. The profits are $\Pi_{\text {in } / \text { out }}=\sum_{l} \sum k H(l) P(k) p(k, l) x(k, l)$. Taking the derivative of $\Pi_{\text {in } / \text { out }}$ for arbitrary out-degree $x \in D$ and in-degree $y \in D$, we obtain

$$
\frac{\partial \Pi_{\text {in } / \text { out }}}{\partial p(x, y)}=0 \Longleftrightarrow p^{*}(x, y)=\frac{1}{2}\left[1+\frac{\gamma}{1-\gamma \hat{k}}\left(\left(1-\bar{p}^{*}\right) x-\phi y\right)\right],
$$

where $\phi=\frac{1}{\hat{k}} \sum_{l} \sum_{k} H(l) P(k) k p^{*}(k, l)$. Using the expression for $p(x, y)$ and the definition of $\bar{p}$ we obtain that

$$
\bar{p}^{*}=\frac{1}{2}\left[1+\frac{\gamma}{1-\gamma \hat{k}}\left(\left(1-\bar{p}^{*}\right) \hat{k}-\phi \frac{\sigma_{l}^{2}+\hat{k}^{2}}{\hat{k}}\right)\right] .
$$

Similarly, using the expression for $p^{*}(x, y)$ and the definition of $\phi$ we obtain

$$
\phi=\frac{1}{2}\left[1+\frac{\gamma}{1-\gamma \hat{k}}\left(\left(1-\bar{p}^{*}\right) \frac{\sigma_{k}^{2}+\hat{k}^{2}}{\hat{k}}-\phi \hat{k}\right)\right] .
$$

Solving the two equations for $\phi$ and for $\bar{p}^{*}$, and plugging these two expressions in the expression for $p^{*}(k, l)$, we obtain that the optimal price charged to a consumer with out-degree $k \in D$ and in-degree $l \in D$ is

$$
p_{\text {in } / \text { out }}(k, l)=\frac{1}{2}+\frac{\gamma \hat{k}}{2} \frac{\left(\gamma \sigma_{l}^{2}+2 \hat{k}\right) k-\left(\gamma \sigma_{k}^{2}+2 \hat{k}\right) l}{\hat{k}^{2}\left(4-4 \gamma \hat{k}-\gamma^{2}\left(\sigma_{k}^{2}+\sigma_{l}^{2}\right)\right)-\gamma^{2} \sigma_{k}^{2} \sigma_{l}^{2}} .
$$


The above analysis, in combination with Proposition A11 completes the proof of Proposition 3 ॥

Proof of Corollary 1. This follows by simple inspection of the expressions of equilibrium consumption in Proposition $3 \|$

Proof of Proposition 4. The expressions of equilibrium consumption in Proposition 4 follow by plugging the expressions of optimal price in Proposition 3 in the expression of the equilibrium demand (expression 2.3). $\|$

Proof of Corollary 2, This follows by simple inspection of the expressions of equilibrium consumption in Proposition $4 \|$

\section{Proof of Proposition 5 .}

Part 1: Consider a consumer with out-degree $k$ (and any in-degree). The additional consumption of the consumer from the move from no discrimination to discrimination on out-degree is

$$
\frac{2+\gamma(k-\hat{k})}{4-4 \gamma \hat{k}-\gamma^{2} \sigma_{k}^{2}}-\frac{1-\gamma \hat{k}+\gamma k}{2-2 \gamma \hat{k}}
$$

Some rearranging leads to the following equality

$$
\operatorname{sign}\left\{\frac{2+\gamma(k-\hat{k})}{4-4 \gamma \hat{k}-\gamma^{2} \sigma_{k}^{2}}-\frac{1-\gamma \hat{k}+\gamma k}{2-2 \gamma \hat{k}}\right\}=\operatorname{sign}\left\{-(k-\hat{k})\left(2-2 \hat{k} \gamma-\gamma^{2} \sigma_{k}^{2}\right)+\sigma_{k}^{2} \gamma\right\} .
$$

Note next that

$$
\frac{\partial}{\partial k}\left(-(k-\hat{k})\left(2-2 \hat{k} \gamma-\gamma^{2} \sigma_{k}^{2}\right)+\sigma_{k}^{2} \gamma\right)=-\left(2-2 \hat{k} \gamma-\sigma_{k}^{2} \gamma^{2}-2\right)<0,
$$

where the inequality follows because $\gamma<1 /\left(2 k^{\max }\right)$ and the variance can be bounded above by $\left(k^{\max }\right)^{2}-\hat{k}^{2}$. To proof of part 1 then follows by evaluating $\operatorname{sign}\left\{-(k-\hat{k})\left(2-2 \hat{k} \gamma-\gamma^{2} \sigma_{k}^{2}\right)+\sigma_{k}^{2} \gamma\right\}$ for $k=\hat{k}$ and by noting that

$$
\frac{\partial}{\partial \sigma_{k}^{2}}\left(-(k-\hat{k})\left(2-2 \hat{k} \gamma-\gamma^{2} \sigma_{k}^{2}\right)+\sigma_{k}^{2} \gamma\right)>0
$$

Part 2: Consider a consumer with out-degree $k$ and in-degree $l$. The additional consumption of the consumer from the move from no discrimination to discrimination of in-degree is

$$
1-\frac{2-\gamma \hat{k}-\gamma l}{4-4 \gamma \hat{k}-\gamma^{2} \sigma_{l}^{2}}+\frac{\gamma\left(2 \hat{k}+\gamma \sigma_{l}^{2}\right)}{\hat{k}\left(4-4 \gamma \hat{k}-\gamma^{2} \sigma_{l}^{2}\right)} k-\frac{1}{2}\left(1+\frac{\gamma k}{1-\gamma \hat{k}}\right) .
$$

Some rearranging leads to the following equality

$$
\begin{aligned}
& \operatorname{sign}\left\{1-\frac{2-\gamma \hat{k}-\gamma l}{4-4 \gamma \hat{k}-\gamma^{2} \sigma_{l}^{2}}+\frac{\gamma\left(2 \hat{k}+\gamma \sigma_{l}^{2}\right)}{\hat{k}\left(4-4 \gamma \hat{k}-\gamma^{2} \sigma_{l}^{2}\right)} k-\frac{1}{2}\left(1+\frac{\gamma k}{1-\gamma \hat{k}}\right)\right\} \\
= & \operatorname{sign}\left\{(1-\gamma \hat{k})\left[2 \hat{k}(l-\hat{k})+\gamma \sigma_{l}^{2}(k-\hat{k})\right]+k \gamma \sigma_{l}^{2}\right\} \equiv L .
\end{aligned}
$$

The proof of part 2 then follows by noticing that $\frac{\partial L}{\partial k}=\sigma_{l}^{2} \gamma(2-\gamma \hat{k})>0$ and $\frac{\partial L}{\partial l}=2 \hat{k}(1-\hat{k} \gamma)>0 . \|$

\section{Proof of Proposition 6 .}

Part 1. The additional aggregate consumer surplus from discriminating on in-degree relative to no 
discrimination is

$$
\begin{aligned}
\Delta C S & =\frac{1}{2} \sum H(l) P(k)\left(\left(1-\frac{2-\gamma \hat{k}-\gamma l}{4-4 \gamma \hat{k}-\gamma^{2} \sigma_{l}^{2}}+\frac{\gamma\left(2 \hat{k}+\gamma \sigma_{l}^{2}\right)}{\hat{k}\left(4-4 \gamma \hat{k}-\gamma^{2} \sigma_{l}^{2}\right)} k\right)^{2}-\left(\frac{1}{2}\left(1+\frac{\gamma k}{1-\gamma \hat{k}}\right)\right)^{2}\right) \\
& =\frac{\gamma^{2} \sigma_{l}^{2}}{8 \hat{k}^{2}} \frac{4 \hat{k}^{4} \gamma^{2}+8 \hat{k}^{3} \sigma_{k}^{2} \gamma^{3}-16 \hat{k}^{3} \gamma+3 \hat{k}^{2} \sigma_{l}^{2} \sigma_{k}^{2} \gamma^{4}-\hat{k}^{2} \sigma_{l}^{2} \gamma^{2}-24 \hat{k}^{2} \sigma_{k}^{2} \gamma^{2}+12 \hat{k}^{2}-8 \hat{k} \sigma_{l}^{2} \sigma_{k}^{2} \gamma^{3}}{(1-\hat{k} \gamma)^{2}\left(4-4 \hat{k} \gamma-\sigma_{l}^{2} \gamma^{2}\right)^{2}} \\
& +\frac{\gamma^{2} \sigma_{l}^{2}}{8 \hat{k}^{2}} \frac{16 \hat{k} \sigma_{k}^{2} \gamma+4 \sigma_{l}^{2} \sigma_{k}^{2} \gamma^{2}}{(1-\hat{k} \gamma)^{2}\left(4-4 \hat{k} \gamma-\sigma_{l}^{2} \gamma^{2}\right)^{2}},
\end{aligned}
$$

which has the same sign as

$$
\begin{aligned}
& 4 \hat{k}^{4} \gamma^{2}+8 \hat{k}^{3} \sigma_{k}^{2} \gamma^{3}-16 \hat{k}^{3} \gamma+3 \hat{k}^{2} \sigma_{l}^{2} \sigma_{k}^{2} \gamma^{4}-\hat{k}^{2} \sigma_{l}^{2} \gamma^{2}-24 \hat{k}^{2} \sigma_{k}^{2} \gamma^{2}+12 \hat{k}^{2}-8 \hat{k} \sigma_{l}^{2} \sigma_{k}^{2} \gamma^{3}+16 \hat{k} \sigma_{k}^{2} \gamma+4 \sigma_{l}^{2} \sigma_{k}^{2} \gamma^{2} \\
= & 4 \hat{k}^{4} \gamma^{2}-16 \hat{k}^{3} \gamma-\hat{k}^{2} \sigma_{l}^{2} \gamma^{2}+12 \hat{k}^{2}+\gamma \sigma_{k}^{2}\left(16 \hat{k}-24 \hat{k}^{2} \gamma-8 \hat{k} \sigma_{l}^{2} \gamma^{2}+8 \hat{k}^{3} \gamma^{2}+3 \hat{k}^{2} \sigma_{l}^{2} \gamma^{3}+4 \sigma_{l}^{2} \gamma\right) \\
> & 4 \hat{k}^{4} \gamma^{2}-8 \hat{k}^{2}-0.25 \hat{k}^{2}+12 \hat{k}^{2}+\gamma \sigma_{k}^{2}\left(16 \hat{k}-12 \hat{k}-2 \hat{k}+8 \hat{k}^{3} \gamma^{2}+3 \hat{k}^{2} \sigma_{l}^{2} \gamma^{3}+4 \sigma_{l}^{2} \gamma\right)>0,
\end{aligned}
$$

where the second to last inequality follows repeated applications of $\gamma k^{\max }<1 / 2$.

Part 2. The additional aggregate consumer surplus from discriminating on out-degree relative to no discrimination is

$$
\begin{aligned}
\Delta C S & =\frac{1}{2} \sum H(l) P(k)\left(\left(\frac{2+\gamma(k-\hat{k})}{4-4 \gamma \hat{k}-\gamma^{2} \sigma_{k}^{2}}\right)^{2}-\left(\frac{1}{2}\left(1+\frac{\gamma k}{1-\gamma \hat{k}}\right)\right)^{2}\right) \\
& =\frac{4(1-\gamma \hat{k})^{2}\left(4+\gamma^{2} \sigma_{k}^{2}\right)-\left(4-4 \gamma \hat{k}-\gamma^{2} \sigma_{k}^{2}\right)^{2}\left(1+\gamma^{2} \sigma_{k}^{2}\right)}{8\left(4-4 \gamma \hat{k}-\gamma^{2} \sigma_{k}^{2}\right)^{2}(1-\gamma \hat{k})^{2}},
\end{aligned}
$$

which has the same sign as

$$
\begin{aligned}
& 4(1-\gamma \hat{k})^{2}\left(4+\gamma^{2} \sigma_{k}^{2}\right)-\left(4-4 \gamma \hat{k}-\gamma^{2} \sigma_{k}^{2}\right)^{2}\left(1+\gamma^{2} \sigma_{k}^{2}\right) \\
= & -\sigma_{k}^{2} \gamma^{2}\left(12 \hat{k}^{2} \gamma^{2}+8 \hat{k} \sigma_{k}^{2} \gamma^{3}-16 \hat{k} \gamma+\sigma_{k}^{4} \gamma^{4}-7 \sigma_{k}^{2} \gamma^{2}+4\right),
\end{aligned}
$$

which in turn has the same sign as

$$
\kappa=-4-12 \hat{k}^{2} \gamma^{2}+16 \hat{k} \gamma-8 \hat{k} \sigma_{k}^{2} \gamma^{3}-\sigma_{k}^{4} \gamma^{4}+7 \sigma_{k}^{2} \gamma^{2} .
$$

The proof is then completed by noting that $\frac{\partial \kappa}{\partial \sigma_{k}^{2}}=-8 \hat{k} \gamma^{3}-2 \sigma_{k}^{2} \gamma^{4}+7 \gamma^{2}>0, \frac{\partial \kappa}{\partial \hat{k}}=$ $\gamma\left(-24 \hat{k} \gamma+16-8 \sigma_{k}^{2} \gamma^{2}\right)>0, \kappa(\hat{k}=0)<0$,

$$
\begin{aligned}
\frac{\partial \kappa}{\partial \gamma} & =-24 \hat{k}^{2} \gamma+16 \hat{k}-24 \hat{k} \sigma_{k}^{2} \gamma^{2}-4 \sigma_{k}^{4} \gamma^{3}+14 \sigma_{k}^{2} \gamma \\
& >16 \hat{k}-12 \hat{k}+\sigma_{k}^{2} \gamma\left(14-24 \hat{k} \gamma-4 \sigma_{k}^{2} \gamma^{2}\right)>0,
\end{aligned}
$$

and that there exists networks for which $\kappa>0$ (e.g., a network in which 0.7 of the population has out degree 9 and 0.3 of the population has out-degree 10 , with $\gamma=0.04) . \|$

Proof of Proposition 7. In the case of price discrimination on out-degree, $\Pi_{\text {out }}=$ $\sum_{k} p_{\text {out }}(k) x_{\text {out }}(k)$, where $p_{\text {out }}(k)$ is in Proposition 3 and $x_{\text {out }}(k)$ is in Proposition 4 Developing we obtain $\Pi_{\text {out }}=1 /\left(4-4 \gamma \hat{k}-\gamma^{2} \sigma_{k}^{2}\right)$. In the case of price discrimination on in-degree, $\Pi_{\text {in }}=$ $\sum_{k} \sum_{l} P(k) H(l) p_{i n}(l) x_{i n}(k, l)$, where $p_{i n}(l)$ is in Proposition 3 and $x_{i n}(k, l)$ is in Proposition 4 Developing we obtain $\Pi_{i n}=1 /\left(4-4 \gamma \hat{k}-\gamma^{2} \sigma_{l}^{2}\right)$. Finally, in the case of price discrimination on indegree and out-degree, the profit of the monopoly is $\Pi_{\text {in } / \text { out }}=\sum_{k} \sum_{l} p_{\text {in } / \text { out }}(k, l) x_{\text {in } / \text { out }}(k, l)$, where $p_{\text {in } / \text { out }}(k, l)$ is in Proposition 3 and $x_{\text {in } / \text { out }}(k, l)$ is in Proposition 4 Using $p_{\text {in } / \text { out }}(k, l)$ and $x_{\text {in } / \text { out }}(k, l)$ 
we can derive the optimal profit:

$$
\Pi_{\text {in } / \text { out }}=\frac{1}{4} \frac{4 \hat{k}^{2}-\gamma^{2} \sigma_{k}^{2} \sigma_{l}^{2}}{4 \hat{k}^{2}(1-\gamma \hat{k})-\gamma^{2} \hat{k}^{2}\left(\sigma_{k}^{2}+\sigma_{l}^{2}\right)-\gamma^{2} \sigma_{l}^{2} \sigma_{k}^{2}} .
$$

$\|$

Proof of Corollary 3 . The results follow from simple inspection of the profits expressions in Proposition 7 for the three regimes of price discrimination. ॥

Proof of Corollary 4, Note that

$$
\Pi_{\text {out }}-\Pi_{\text {uniform }}=\frac{1}{4} \frac{\gamma^{2} \sigma_{k}^{2}}{\left(4-4 \gamma \hat{k}-\gamma^{2} \sigma_{k}^{2}\right)(1-\gamma \hat{k})},
$$

is increasing in $\sigma_{k}^{2}$ and $\hat{k}$. Similarly

$$
\Pi_{\text {in }}-\Pi_{\text {uniform }}=\frac{1}{4} \frac{\gamma^{2} \sigma_{x}^{2}}{\left(4-4 \gamma \hat{k}-\gamma^{2} \sigma_{x}^{2}\right)(1-\gamma \hat{k})},
$$

is increasing in $\sigma_{l}^{2}$ and $\hat{k}$. Comparing we obtain that the monopoly prefers to learn the out-degree than the in-degree if and only if $\sigma_{k}^{2}>\sigma_{l}^{2}$. Next, the value of the monopoly for learning both the in-degree and the out-degree relative of no-information is

$$
\Pi_{\text {in } / \text { out }}-\Pi_{\text {uniform }}=\frac{1}{4} \frac{\gamma^{2} \hat{k}\left[\hat{k}\left(\sigma_{l}^{2}+\sigma_{k}^{2}\right)+\gamma \sigma_{l}^{2} \sigma_{k}^{2}\right]}{(1-\gamma \hat{k})\left[\hat{k}^{2}\left(4-4 \hat{k} \gamma-\gamma^{2}\left(\sigma_{k}^{2}+\sigma_{l}^{2}\right)\right)-\gamma^{2} \sigma_{l}^{2} \sigma_{k}^{2}\right]},
$$

which is increasing in the variance of the in-degree and the variance of the out-degree.\|

Proof of Corollary 5. We say that the value for the monopoly from learning the out-degrees and in-degrees of all consumers exhibits complementarity if

$$
\Pi_{\text {in } / \text { out }}-\Pi_{\text {out }} \geq \Pi_{\text {in }}-\Pi_{\text {uniform }}
$$

We can re-write A9 as follows

$$
\frac{1}{4} \frac{4 \hat{k}^{2}-\gamma^{2} \sigma_{k}^{2} \sigma_{l}^{2}}{\hat{k}^{2}\left[4-4 \gamma \hat{k}-\gamma^{2}\left(\sigma_{k}^{2}+\sigma_{l}^{2}\right)\right]-\gamma^{2} \sigma_{k}^{2} \sigma_{l}^{2}}-\frac{1}{4-4 \gamma \hat{k}-\gamma^{2} \sigma_{k}^{2}} \geq \frac{1}{4-4 \gamma \hat{k}-\gamma^{2} \sigma_{l}^{2}}-\frac{1}{4} \frac{1}{1-\gamma \hat{k}}
$$

or equivalently

$$
\hat{k}\left(8-8 \hat{k} \gamma-3 \sigma_{k}^{2} \gamma^{2}-3 \sigma_{l}^{2} \gamma^{2}\right)+\gamma \sigma_{k}^{2}\left(2-\sigma_{l}^{2} \gamma^{2}\right)+2 \sigma_{l}^{2} \gamma \geq 0
$$

which holds for the entire range of parameters.||

In this last part of the Appendix we prove the results under correlation between in-degree and out-degree, i.e., Proposition 8 and Proposition 9 We derive these results imposing that the correlation satisfies Assumption 2 and $\sigma_{k}^{2}=\sigma_{l}^{2}$. In online Appendix II we derive the optimal pricing strategy under the three different regimes of price discrimination for general correlation between in-degrees and outdegrees ${ }^{28}$ In that online Appendix we also compute equilibrium consumption at the optimal price. In order to avoid repetition in the derivations, in the proof of Proposition 8 and Proposition 9 we introduce the expressions of optimal pricing and of equilibrium consumption at the optimal price derived for the general model, and we then use Assumption 2 to specialize these expressions. We refer the interested reader to online Appendix II for the derivation of these expressions.

In the general case, the correlation between in- and out-degrees is captured by

$$
\rho=\frac{\operatorname{Cov}(k, l)}{\sigma_{k} \sigma_{l}}=\frac{E[(k-\hat{k})(l-\hat{k})]}{\sigma_{k} \sigma_{l}}=\frac{E[k l]-\hat{k}^{2}}{\sigma_{k} \sigma_{l}}=\frac{\bar{k}-\hat{k}}{\sigma_{k} \sigma_{l}} \hat{k},
$$

where recall that $\bar{k} \equiv \sum_{l} \bar{H}(l) \sum_{k} P(k \mid l) k$. The case of perfect positive correlation occurs when $\rho=1$, which when $\sigma_{l}=\sigma_{k}=\sigma$ implies that $\bar{k}=\frac{\sigma^{2}+\hat{k}^{2}}{\hat{k}}$.

28. The derivation mirrors the steps we have developed in the derivation of the optimal pricing under the assumption of independence between in-degrees and out-degrees. 
Furthermore, as we shall see the optimal pricing function under correlation depends on other statistics of the network, which we now introduce. We denote by $\hat{k}(l)=\sum_{k} P(k \mid l) k$ the average out-degree of a consumer conditional on the consumer's in-degree $l$; the average out-degree of a consumer conditional on the consumer's in-degree $l$ is a random variable and we denote by $\sigma_{\hat{k}(l)}^{2}=\sum_{l} H(l)[\hat{k}-\hat{k}(l)]^{2}$ its variance. Similarly, we define $\hat{l}(k)=\sum_{l} H(l \mid k) l$ and $\sigma_{\hat{l}(k)}^{2}=\sum_{k} P(k)[\hat{k}-\hat{l}(k)]^{2}$. Assumption 2 and $\sigma_{l}^{2}=\sigma_{k}^{2}=\sigma^{2}$ imply that $\hat{l}(k)=\hat{k}+\frac{\hat{k}(\bar{k}-\hat{k})(k-\hat{k})}{\sigma_{k}^{2}}, \hat{k}(l)=\hat{k}+\frac{\hat{k}(\bar{k}-\hat{k})(l-\hat{k})}{\sigma^{2}}, \sigma_{\hat{l}(k)}^{2}=\sigma_{\hat{k}(l)}^{2}=\rho^{2} \sigma^{2}$.

\section{Proof of Proposition 8}

Uniform price. Under general correlation it is possible to show that optimal price is $p=1 / 2$, equilibrium demand at the optimal price is $x(k, l)=x(k)=\frac{1}{2}\left(1+\frac{\gamma k}{1-\gamma \bar{k}}\right)$ and so the monopoly profits are

$$
\Pi_{\text {uniform }}=\frac{1}{4}\left[1+\frac{\gamma \hat{k}}{1-\gamma \bar{k}}\right]=\frac{\hat{k}-\gamma \rho \sigma^{2}}{4\left(\hat{k}-\gamma \rho \sigma^{2}\right)-4 \gamma \hat{k}^{2}} .
$$

where the second equality uses $\rho=\frac{\bar{k}-\hat{k}}{\sigma^{2}} \hat{k}$. That $\Pi_{\text {uniform }}$ increases in $\rho$ is evident.

Discrimination on out-degree. Consider first the case of price discrimination on out-degree. Under general correlation it is possible to show that the optimal price charged to a consumer with out-degree $k \in D$ and the equilibrium demand at the optimal price of a consumer with out-degree $k \in D$ are:

$$
\begin{aligned}
& p_{\text {out }}^{*}(k)=\frac{1}{2}-\frac{\gamma \hat{k}}{2} \frac{\hat{k}[2-\gamma(\bar{k}-\hat{k})][\hat{l}(k)-k]+\gamma\left[\hat{l}(k) \sigma_{k}^{2}-k \sigma_{\hat{l}(k)}^{2}\right]}{\hat{k}^{2}(2-\bar{k} \gamma)^{2}-\gamma^{2}\left(\sigma_{\hat{l}(k)}^{2}+\hat{k}^{2}\right)\left(\sigma_{k}^{2}+\hat{k}^{2}\right)}, \\
& x_{\text {out }}^{*}(k)=\frac{1}{2}+\frac{\gamma \hat{k}}{2} \frac{\hat{k}[2-\gamma(\bar{k}-\hat{k})][\hat{l}(k)+k]+\gamma\left[\hat{l}(k) \sigma_{k}^{2}+k \sigma_{\hat{l}(k)}^{2}\right]}{\hat{k}^{2}(2-\bar{k} \gamma)^{2}-\gamma^{2}\left(\sigma_{\hat{l}(k)}^{2}+\hat{k}^{2}\right)\left(\sigma_{k}^{2}+\hat{k}^{2}\right)} .
\end{aligned}
$$
follows:

Under Assumption 2 and $\sigma_{l}^{2}=\sigma_{k}^{2}=\sigma^{2}$, we can rewrite expression A10 and expression A11 as

$$
\begin{aligned}
& p_{\text {out }}^{*}(k)=\frac{1}{2}+\frac{1}{2} \frac{(1-\rho) \gamma\left[2(k-\hat{k})\left(\hat{k}-\rho \gamma \sigma^{2}\right)-\gamma \sigma^{2} \hat{k}(1+\rho)\right]}{4\left[\hat{k}-\gamma \rho \sigma^{2}\right]-4 \gamma \hat{k}^{2}-\gamma^{2} \sigma^{2} \hat{k}(1-\rho)^{2}}, \\
& x_{\text {out }}^{*}(k)=\frac{1}{2}+\frac{1}{2} \frac{\hat{k}\left(\gamma^{2} \sigma^{2}-\gamma^{2} \rho \sigma^{2}+2 \gamma \hat{k}\right)(1-\rho)+2 \gamma \hat{k}(1+\rho) k}{4\left(\hat{k}-\gamma \rho \sigma^{2}\right)-4 \gamma \hat{k}^{2}-\gamma^{2} \sigma^{2} \hat{k}(1-\rho)^{2}},
\end{aligned}
$$

and then we can compute expected profits $\Pi_{\text {out }}=\sum_{k} P(k) p(k) x(k)$ and obtain

$$
\Pi_{\text {out }}^{*}=\frac{\hat{k}-\gamma \rho \sigma^{2}}{4\left(\hat{k}-\gamma \rho \sigma^{2}\right)-4 \gamma \hat{k}^{2}-\gamma^{2} \sigma^{2} \hat{k}(1-\rho)^{2}} .
$$

Discrimination on in-degree. We now consider the case of price discrimination on in-degree. Under general correlation it is possible to show that the optimal price charged to a consumer with out-degree $l \in D$ and the equilibrium demand at the optimal price of a consumer with out-degree $k \in D$ and in-degree $l \in D$ are:

$$
\begin{aligned}
p_{i n}^{*}(l) & =\frac{1}{2}+\frac{\gamma \hat{k}}{2} \frac{\hat{k}[2-\gamma(\bar{k}-\hat{k})][\hat{k}(l)-l]+\gamma\left[\hat{k}(l) \sigma_{l}^{2}-l \sigma_{\hat{k}(l)}^{2}\right]}{\hat{k}^{2}(2-\bar{k} \gamma)^{2}-\gamma^{2}\left(\sigma_{\hat{k}(l)}^{2}+\hat{k}^{2}\right)\left(\sigma_{l}^{2}+\hat{k}^{2}\right)}, \\
x_{i n}^{*}(k, l) & =\frac{1}{2}+\frac{\gamma \hat{k}}{2} \frac{\hat{k}[2-\gamma(\bar{k}-\hat{k})][l-\hat{k}(l)+2 k]+\gamma\left[l \sigma_{\hat{k}(l)}^{2}-\hat{k}(l) \sigma_{l}^{2}+2 k \sigma_{l}^{2}\right]}{\hat{k}^{2}(2-\bar{k} \gamma)^{2}-\gamma^{2}\left(\sigma_{\hat{k}(l)}^{2}+\hat{k}^{2}\right)\left(\sigma_{l}^{2}+\hat{k}^{2}\right)} .
\end{aligned}
$$

Under assumption 2 and $\sigma_{l}^{2}=\sigma_{k}^{2}=\sigma^{2}$ we can re-write expression $\mathrm{A} 12$ and expression A13 as 
follows

$$
\begin{aligned}
p_{i n}^{*}(l) & =\frac{1}{2}-\frac{1}{2} \frac{(1-\rho) \gamma\left[2(l-\hat{k})\left(\hat{k}-\rho \gamma \sigma^{2}\right)-\gamma \sigma^{2} \hat{k}(1+\rho)\right]}{4\left[\hat{k}-\gamma \rho \sigma^{2}\right]-4 \gamma \hat{k}^{2}-\gamma^{2} \sigma^{2} \hat{k}(1-\rho)^{2}} \\
x_{i n}^{*}(l, k) & =\frac{1}{2}+\frac{1}{2} \frac{\gamma \hat{k}\left[((l-\hat{k})(1-\rho)+2 k)\left(2 \hat{k}-\rho \sigma^{2} \gamma\right)+\gamma\left(l \rho^{2} \sigma^{2}-\sigma^{2}(\hat{k}+\rho(l-\hat{k}))+2 k \sigma^{2}\right)\right]}{\left[2 \hat{k}-\gamma\left(\rho \sigma^{2}+\hat{k}^{2}\right)\right]^{2}-\gamma^{2}\left(\sigma^{2}+\hat{k}^{2}\right)\left(\rho \sigma^{2}+\hat{k}^{2}\right)} .
\end{aligned}
$$

Computing profits we obtain the same expression in the case of discrimination on out-degree. Hence, the expected profits under discrimination in in-degrees or in out-degrees are the same, given assumption 2 and the assumption that $\sigma_{l}^{2}=\sigma_{k}^{2}$.

We now show that $\Pi_{\text {out }}^{*}$ and $\Pi_{\text {in }}^{*}$ are increasing in $\rho$. First note that we can rewrite $\Pi_{\text {out }}^{*}$ as

$$
\Pi_{\text {out }}^{*}=\frac{1}{4}+\frac{1}{4} \frac{\gamma^{2} \sigma^{2} \hat{k}(1-\rho)^{2}+4 \gamma \hat{k}^{2}}{4\left(\hat{k}-\gamma \rho \sigma^{2}\right)-4 \gamma \hat{k}^{2}-\gamma^{2} \sigma^{2} \hat{k}(1-\rho)^{2}}
$$

and that

$$
\begin{aligned}
\operatorname{sign}\left\{\frac{\partial \Pi_{\text {out }}^{*}}{\partial \rho}\right\} & =\operatorname{sign}\left\{\frac{\partial}{\partial \rho}\left(\frac{\gamma^{2} \sigma^{2} \hat{k}(1-\rho)^{2}+4 \gamma \hat{k}^{2}}{4\left(\hat{k}-\gamma \rho \sigma^{2}\right)-4 \gamma \hat{k}^{2}-\gamma^{2} \sigma^{2} \hat{k}(1-\rho)^{2}}\right)\right\} \\
& =-\operatorname{sign}\left\{\frac{\partial}{\partial \rho}\left(\frac{\hat{k}-\gamma \rho \sigma^{2}}{\gamma^{2} \sigma^{2} \hat{k}(1-\rho)^{2}+4 \gamma \hat{k}^{2}}\right)\right\}
\end{aligned}
$$

and

$$
\operatorname{sign}\left\{\frac{\partial}{\partial \rho}\left(\frac{\hat{k}-\gamma \rho \sigma^{2}}{\gamma^{2} \sigma^{2} \hat{k}(1-\rho)^{2}+4 \gamma \hat{k}^{2}}\right)\right\}=\operatorname{sign}\left\{-\hat{k} \sigma^{2} \gamma^{2}(\rho+1)\left(2 \hat{k}+\sigma^{2} \gamma(1-\rho)\right)\right\}<0 .
$$

Discrimination in/out-degree. We now turn to the case of price discrimination in both dimensions. Again, for general correlation we have that the price charged to a consumer with out-degree $k \in D$ and in-degree $l \in D$ is

$$
p_{\text {in } / \text { out }}^{*}(k, l)=\frac{1}{2}+\frac{1}{2} \frac{\gamma \hat{k}^{2}[\gamma(\bar{k}-\hat{k})-2][l-k]+\gamma^{2} \hat{k}\left[k \sigma_{l}^{2}-l \sigma_{k}^{2}\right]}{\hat{k}^{2}(2-\bar{k} \gamma)^{2}-\gamma^{2}\left(\sigma_{k}^{2}+\hat{k}^{2}\right)\left(\sigma_{l}^{2}+\hat{k}^{2}\right)} .
$$

and that the monopoly profits at the optimal price

$$
\Pi_{\text {in } / \text { out }}=\sum_{k} P(k) \sum_{l} H(l \mid k) p_{\text {in } / \text { out }}(k, l) x_{\text {in } / \text { out }}(k, l)
$$

can be written as

$$
\Pi_{\text {in } / \text { out }}=\sum_{k} \sum_{l} H(l \mid k) p_{\text {in } / \text { out }}(k, l)^{2}+\frac{\gamma \phi \bar{p} \hat{k}}{1-\gamma \bar{k}}
$$

where $\phi=\frac{1}{\hat{k}} \sum_{l} H(l) \sum_{k} P(k \mid l) k p_{\text {in } / \text { out }}(k, l)$ and $\bar{p}=\frac{1}{\hat{k}} \sum_{l} H(l) \sum_{k} P(k \mid l) l p_{\text {in } / \text { out }}(k, l)$.

Using Assumption 2 and the assumption that $\sigma_{l}^{2}=\sigma_{k}^{2}$ we rewrite expression A14 as follows

$$
\begin{aligned}
p_{\text {in } / \text { out }}(k, l) & =\frac{1}{2}+\frac{1}{2} \frac{\gamma \hat{k}\left[\gamma \sigma^{2}(1-\rho)+2 \hat{k}\right]}{\left[2 \hat{k}-\gamma\left(\rho \sigma^{2}+\hat{k}^{2}\right)\right]^{2}-\gamma^{2}\left(\sigma^{2}+\hat{k}^{2}\right)^{2}}[k-l] \\
& =\frac{1}{2}+\frac{1}{2} A[k-l],
\end{aligned}
$$

where

$$
A=\frac{\gamma \hat{k}\left[\gamma \sigma^{2}(1-\rho)+2 \hat{k}\right]}{\left[2 \hat{k}-\gamma\left(\rho \sigma^{2}+\hat{k}^{2}\right)\right]^{2}-\gamma^{2}\left(\sigma^{2}+\hat{k}^{2}\right)^{2}},
$$

We can then write explicitly $\phi$ and $\bar{p}$ and obtain that

$$
\phi=\frac{1}{\hat{k}} \sum_{l} H(l) \sum_{k} P(k \mid l) k p_{\text {in } / \text { out }}(k, l)=\frac{1}{2}+\frac{1}{2 \hat{k}} A \sigma^{2}(1-\rho)
$$




$$
\bar{p}=\frac{1}{\hat{k}} \sum_{l} H(l) \sum_{k} P(k \mid l) l p_{\text {in } / \text { out }}(k, l)=\frac{1}{2}-\frac{1}{2 \hat{k}} A \sigma^{2}(1-\rho) .
$$

Furthermore, we can develop an expression of

$$
\sum_{l} H(l) \sum_{k} P(k \mid l) p_{\text {in } / \text { out }}(k, l)^{2}=\frac{1}{4}+\frac{1}{2} A^{2}\left[\sigma^{2}(1-\rho)\right]^{2} .
$$

Substituting $\bar{p}, \phi$ and $\sum_{l} H(l) \sum_{k} P(k \mid l) p_{\text {in } / o u t}(k, l)^{2}$ in the expression for $\Pi_{\text {in } / o u t}$, we obtain

$$
\Pi_{\text {in } / \text { out }}^{*}=\frac{1}{4} \frac{2 \hat{k}-\gamma \sigma^{2}(1+\rho)}{2 \hat{k}(1-\gamma \hat{k})-\gamma \sigma^{2}(1+\rho)} .
$$

Taking the derivatives of the profit with respect to $\rho$ we obtain

$$
\frac{\partial \Pi_{\text {in } / \text { out }}^{*}}{\partial \rho}=\frac{1}{2} \frac{\gamma^{2} \hat{k}^{2} \sigma^{2}}{\left[2 \hat{k}(1-\gamma \hat{k})-\gamma \sigma^{2}(1+\rho)\right]^{2}}>0
$$

This concludes the proof of Proposition 8

\section{Proof of Proposition 9.}

Part 1. The additional profit that a monopoly gets for being able to discriminate on out-degree relative to charge a uniform price is

$$
\begin{aligned}
\Delta \Pi_{k, 0}(\rho) & =\frac{1}{4}+\frac{1}{4} \frac{\gamma^{2} \sigma^{2} \hat{k}(1-\rho)^{2}+4 \gamma \hat{k}^{2}}{4\left(\hat{k}-\gamma \rho \sigma^{2}\right)-4 \gamma \hat{k}^{2}-\gamma^{2} \sigma^{2} \hat{k}(1-\rho)^{2}}-\left(\frac{1}{4}\left[1+\frac{\gamma \hat{k}}{1-\gamma \bar{k}}\right]\right) \\
& =\frac{\gamma^{2} \sigma^{2} \hat{k}}{4}\left(\frac{(1-\rho)^{2}\left(\hat{k}-\gamma \rho \sigma^{2}\right)}{\left(4 \hat{k}-4 \gamma \rho \sigma^{2}-4 \gamma \hat{k}^{2}-\gamma^{2} \sigma^{2} \hat{k}(1-\rho)^{2}\right)\left(\hat{k}-\gamma \rho \sigma^{2}-\gamma \hat{k}^{2}\right)}\right) .
\end{aligned}
$$

We observe that $\Delta \Pi_{k, 0}(1)=0$ and for any $\rho<1, \Delta \Pi_{k, 0}(\rho) \geq 0$. We now show that $\Delta \Pi_{k, 0}$ is decreasing in $\rho$. To see this note that

$$
\begin{aligned}
\operatorname{sign}\left\{\frac{\partial \Delta \Pi_{k, 0}}{\partial \rho}\right\} & =-\operatorname{sign}\left\{\frac{\partial}{\partial \rho}\left(\frac{\left(4 \hat{k}-4 \gamma \rho \sigma^{2}-4 \gamma \hat{k}^{2}-\gamma^{2} \sigma^{2} \hat{k}(1-\rho)^{2}\right)\left(\hat{k}-\gamma \rho \sigma^{2}-\gamma \hat{k}^{2}\right)}{(1-\rho)^{2}\left(\hat{k}-\gamma \rho \sigma^{2}\right)}\right)\right\} \\
& =-\operatorname{sign}\left\{\frac{\partial}{\partial \rho}\left(4 \frac{\left(\hat{k}-\gamma \rho \sigma^{2}-\gamma \hat{k}^{2}\right)^{2}}{(1-\rho)^{2}\left(\hat{k}-\gamma \rho \sigma^{2}\right)}+\frac{\gamma^{3} \sigma^{2} \hat{k}^{3}}{\hat{k}-\gamma \rho \sigma^{2}}\right)\right\} .
\end{aligned}
$$

Next, $\frac{\partial}{\partial \rho}\left(\frac{\gamma^{3} \sigma^{2} \hat{k}^{3}}{\hat{k}-\gamma \rho \sigma^{2}}\right) \geq 0$, and

$$
\operatorname{sign}\left\{\frac{\partial}{\partial \rho}\left(\frac{\left(\hat{k}-\gamma \rho \sigma^{2}-\gamma \hat{k}^{2}\right)^{2}}{(1-\rho)^{2}\left(\hat{k}-\gamma \rho \sigma^{2}\right)}\right)\right\}=\operatorname{sign}\{\xi(\rho)\},
$$

where

$\xi(\rho)=-2 \gamma \sigma^{2}(1-\rho)\left(\hat{k}-\gamma \rho \sigma^{2}\right)+2\left(\hat{k}-\gamma \rho \sigma^{2}\right)\left(\hat{k}-\gamma \rho \sigma^{2}-\gamma \hat{k}^{2}\right)+\gamma \sigma^{2}(1-\rho)\left(\hat{k}-\gamma \rho \sigma^{2}-\gamma \hat{k}^{2}\right)$.

Since $\frac{\partial \xi}{\partial \rho}=\sigma^{2} \gamma\left(3 \hat{k}^{2} \gamma-3 \hat{k}+\sigma^{2} \gamma+2 \sigma^{2} \gamma \rho\right) \leq 0$ and $\xi(1)=2\left(\hat{k}-\gamma \sigma^{2}\right)\left(\hat{k}-\gamma \sigma^{2}-\gamma \hat{k}^{2}\right) \geq 0$, it follows that $\xi(\rho) \geq 0$. This proves the claim that $\Delta \Pi_{k, 0}$ is decreasing in $\rho$. The proof for the case of discrimination on in-degree is identical.

Part 2. We now prove the second part of Proposition 9 By definition, the value for the monopoly from learning out-degrees and in-degrees of all consumers exhibits complementarity iff $\Pi_{\text {in } / \text { out }}^{*}-\Pi_{\text {out }}^{*} \geq$ 


$$
\begin{aligned}
& \Pi_{\text {in }}^{*}-\Pi_{\text {uniform }}^{*}, \text { i.e., iff } \\
& \qquad \begin{aligned}
0 & \leq \frac{1}{4} \frac{2 \hat{k}-\gamma \sigma^{2}(1+\rho)}{2 \hat{k}(1-\gamma \hat{k})-\gamma \sigma^{2}(1+\rho)}-2 \frac{\hat{k}-\gamma \rho \sigma^{2}}{4\left(\hat{k}-\gamma \rho \sigma^{2}\right)-4 \gamma \hat{k}^{2}-\gamma^{2} \sigma^{2} \hat{k}(1-\rho)^{2}}+\frac{\hat{k}-\gamma \rho \sigma^{2}}{4\left(\hat{k}-\gamma \rho \sigma^{2}\right)-4 \gamma \hat{k}^{2}} \\
& =\frac{-\hat{k} \sigma^{2} \gamma^{2}(1-\rho) \eta(\rho)}{4\left(-\gamma \hat{k}^{2}-\gamma \rho \sigma^{2}+\hat{k}\right)\left(-2 \gamma \hat{k}^{2}-\gamma \rho \sigma^{2}-\gamma \sigma^{2}+2 \hat{k}\right)\left(-\gamma^{2} \hat{k} \rho^{2} \sigma^{2}+2 \gamma^{2} \hat{k} \rho \sigma^{2}-\gamma^{2} \hat{k} \sigma^{2}-4 \gamma \hat{k}^{2}-4 \gamma \rho \sigma^{2}+4 \hat{k}\right)}
\end{aligned}
\end{aligned}
$$

where

$$
\eta(\rho)=4 \hat{k}^{3} \gamma \rho-3 \hat{k}^{2} \sigma^{2} \gamma^{2} \rho^{2}+2 \hat{k}^{2} \sigma^{2} \gamma^{2} \rho+\hat{k}^{2} \sigma^{2} \gamma^{2}-4 \hat{k}^{2} \rho+6 \hat{k} \sigma^{2} \gamma \rho^{2}-2 \hat{k} \sigma^{2} \gamma-2 \sigma^{4} \gamma^{2} \rho^{3}+2 \sigma^{4} \gamma^{2} \rho
$$

The following claim is sufficient to establish the desired result.

Claim 1. The following holds

a. $\hat{k}-\gamma \hat{k}^{2}-\gamma \rho \sigma^{2}>0$.

b. $2 \hat{k}-2 \gamma \hat{k}^{2}-\gamma \sigma^{2}-\gamma \rho \sigma^{2}>0$.

c. $4 \hat{k}-4 \gamma \hat{k}^{2}-\gamma^{2} \hat{k} \sigma^{2}-4 \gamma \rho \sigma^{2}+2 \gamma^{2} \hat{k} \rho \sigma^{2}-\gamma^{2} \hat{k} \rho^{2} \sigma^{2}>0$.

d. $\eta(\rho)$ changes from positive to negative at a unique $\rho<0$.

Proof of Claim 1. To see part (a) note that

$$
\operatorname{sign}\left\{\hat{k}-\gamma \hat{k}^{2}-\gamma \rho \sigma^{2}\right\}=\operatorname{sign}\left\{1-\frac{1}{\hat{k}} \gamma \hat{k}^{2}-\frac{1}{\hat{k}} \gamma \rho \sigma^{2}\right\}>0,
$$

where the inequality follows because the assumption $\gamma<\frac{1}{2 k^{\max }}$ implies that

$$
1-\frac{1}{\hat{k}} \gamma \hat{k}^{2}-\frac{1}{\hat{k}} \gamma \rho \sigma^{2}>1-\frac{1}{2 \hat{k} k^{\max }} \hat{k}^{2}-\frac{1}{2 \hat{k} k^{\max }} \rho \sigma^{2}>\frac{1}{2}-\frac{1}{2} \frac{\sigma^{2}}{\hat{k} k^{\max }}>0,
$$

where the last inequality follows because $\hat{k} k^{\max } \geq \hat{k}\left(k^{\max }-\hat{k}\right)=\sum P(k) k k^{\max }-\hat{k}^{2} \geq \sum P(k) k^{2}-$ $\hat{k}^{2}=\sigma^{2}$. Furthermore, part (b) follows from part (a).

To see part (c), note that $4 \hat{k}-4 \gamma \hat{k}^{2}-\gamma^{2} \hat{k} \sigma^{2}-4 \gamma \rho \sigma^{2}+2 \gamma^{2} \hat{k} \rho \sigma^{2}-\gamma^{2} \hat{k} \rho^{2} \sigma^{2}$ is decreasing in $\rho$ and therefore,

$$
\begin{aligned}
4 \hat{k}-4 \gamma \hat{k}^{2}-\gamma^{2} \hat{k} \sigma^{2}-4 \gamma \rho \sigma^{2}+2 \gamma^{2} \hat{k} \rho \sigma^{2}-\gamma^{2} \hat{k} \rho^{2} \sigma^{2} & >4 \hat{k}-4 \gamma \hat{k}^{2}-\gamma^{2} \hat{k} \sigma^{2}-4 \gamma \sigma^{2}+2 \gamma^{2} \hat{k} \sigma^{2}-\gamma^{2} \hat{k} \sigma^{2} \\
& =4\left(\hat{k}-\gamma \hat{k}^{2}-\gamma \sigma^{2}\right)>0
\end{aligned}
$$

where the last inequality follows from part (a). Finally, to prove part (d), it sufficient to show the following: (1.) $\eta(-1)>0$, (2.) $\eta(0)<0$, (3.) $\eta(1) \leq 0$ and (4.) $\frac{d^{2} \eta}{d \rho^{2}}>0$ for $\rho \in[-1,1]$. Point 1,2 , and 3 are immediate from substituting $\rho=-1,0,1$ in $\eta(\rho)$ respectively and applying part a. Finally, to show point 4 note that

$$
\begin{aligned}
\frac{d^{2} \eta}{d \rho^{2}} & =-6 \hat{k}^{2} \sigma^{2} \gamma^{2}+12 \hat{k} \sigma^{2} \gamma-12 \sigma^{4} \gamma^{2} \rho \\
& >-6 \hat{k}^{2} \sigma^{2} \gamma^{2}+12 \hat{k} \sigma^{2} \gamma-12 \sigma^{4} \gamma^{2}=6 \gamma \sigma^{2}\left(2 \hat{k}-\hat{k}^{2} \gamma-2 \sigma^{2} \gamma\right)>0,
\end{aligned}
$$

where the proof for the last inequality follows from part (a). ॥

\section{Supplementary Data}

Supplementary materials are available at Review of Economic Studies online.

Acknowledgements. A previous version of the paper was circulated under the title "The Value of Network Information". We thank an Editor and three Referees for their comments. We thank Matt 
Jackson, Shachar Kariv, Jacob Leshno, Markus Mobius, and participants to seminars at UC Berkeley, Stanford University, Brown University, Microsoft Research New England, Tel Aviv University, Ohio State University, CalTech, Washington University at St. Louis, University of Toronto, UCLA, UCSD, UPenn, and The University of Essex, for valuable comments. We thank Neil Thakral, Michael Thaler, and Stephen Nei for excellent research assistance. Itay Fainmesser is greatful to the Economics Department at Stanford University for their hospitality and financial support during the academic year 2013-14. Andrea Galeotti is grateful to the European Research Council for support through ERC-starting grant (award no. 283454), to The Leverhulme Trust for support through the Philip Leverhulme Prize, and to the Economics Department of the European Institute for their hospitality during the academic year 2014-15.

\section{REFERENCES}

Aral, S. and D. Walker (2012). Identifying Influential and Susceptible Members of Social Networks. Science 337(6092), 337-341.

Arthur, D., R. Motwani, A. Sharma, and Y. Xu (2009). Pricing Strategies for Viral Marketing on Social Networks. Mimeo. Department of Computer Science, Stanford University.

Ballester, C., A. Calvó-Armengol, and Y. Zenou (2006). Who's Who in Networks. Wanted: The Key Player. Econometrica 74(5), 1403-1417.

Bapna, R. and A. Umyarov (2014). Do Your Online Friends Make You Pay? A Randomized Field Experiment in an Online Music Social Network. Management Science, Forthcoming.

Bearden, W. O. and M. J. Etzel (1982). Reference Group Influence on Product and Brand Purchase Decisions. Journal of Consumer Research, 183-194.

Bernstein, S. and E. Winter (2012). Contracting with Heterogeneous Externalities. American Economic Journal: Microeconomics 4(2), 50-76.

Bloch, F. and N. Quérou (2013). Pricing in Social Networks. Games and Economic Behavior 80, $243-261$.

Bramoullé, Y., R. Kranton, and M. D'Amours (2014). Strategic Interaction and Networks. American Economic Review 104(3), 898-930.

Campbell, A. (2013). Word-of-mouth Communication and Percolation in Social Networks. American Economic Review 103(6), 2466-2498.

Candogan, O., K. Bimpikis, and A. Ozdaglar (2012). Optimal Pricing in Networks With Externalities. Operations Research 60(4), 883-905.

Domingos, P. and M. Richardson (2001). Mining the Network Value of Customers. In Proceedings of the 7th International Conference on Knowledge Discovery and Data Mining, pp. 57-66. ACM.

Fainmesser, I. P. and D. A. Goldberg (2012). Cooperation in Partly Observable Networked Markets. Working Paper.

Farrell, J. and G. Saloner (1985). Standardization, Compatibility, and Innovation. The RAND Journal of Economics 16, 70-83.

Feige, U., A. Fiat, and A. Shamir (1988). Zero-knowledge Proofs of Identity. Journal of Cryptology 1(2), $77-94$.

Froomkin, A. M. (2000). The Death of Privacy? Stanford Law Review 52(5), 1461-1543.

Galeotti, A. and S. Goyal (2009). Influencing the Influencers: A Theory of Strategic Diffusion. The RAND Journal of Economics 40(3), 509-532.

Galeotti, A., S. Goyal, M. Jackson, F. Vega-Redondo, and L. Yariv (2010). Network Games. Review of Economic Studies $77(1), 218-244$.

Glaeser, E. and J. A. Scheinkman (2002). Non-Market Interactions. In L. Hansen and S. Turnovsky (Eds.), Advances in Economics and Econometrics: Theory and Applications. Cambridge University Press.

Goldenberg, J., S. Han, D. R. Lehmann, and J. W. Hong (2009). The Role of Hubs in the Adoption Process. Journal of Marketing 73(2), 1-13.

Goyal, S. and M. Kearns (2012). Competitive Contagion in Networks. STOC.

Green, H. (2008, October). Google: Harnessing the Power of Cliques. Business Week, 50.

Hartline, J., V. Mirrokni, and M. Sundararajan (2008). Optimal Marketing Strategies Over Social Networks. In Proceedings of the 17th International Conference on World Wide Web, Beijing, China, pp. 189-198. ACM.

Johnson, J. P. and D. P. Myatt (2006). On the Simple Economics of Advertising, Marketing, and Product Design. American Economic Review 96(3), 756-784.

Katz, M. L. and C. Shapiro (1985). Network Externalities, Competition, and Compatibility. American Economic Review 75, 424-440.

Kazaz, B., M. Dada, and H. Moskowitz (2005). Global Production Planning Under Exchange-Rate Uncertainty. Management Science 51(7), 1101-1119.

Keller, E., B. Fay, and J. Berry (2007). Leading the Conversation: Influencers' Impact on Word of Mouth and the Brand Conversation. In The Keller Fay Group, Word of Mouth Marketing Research Symposium. 
Kempe, D., J. Kleinberg, and E. Tardos (2003). Maximizing the Spread of Influence Through a Social Network. In Proceedings of the 9th International Conference on Knowledge Discovery and Data Mining, pp. 137-146. ACM.

Krackhardt, D. (1996). Structural Leverage in Marketing. In D. Iacobucci (Ed.), Networks in Marketing, pp. 50-59. SAGE Publications Thousand Oaks/California.

Leskovec, J., L. A. Adamic, and B. A. Huberman (2007). The Dynamics of Viral Marketing. ACM Transactions on the Web (TWEB) 1(1), 5.

Nair, H. S., P. Manchanda, and T. Bhatia (2010). Asymmetric Social Interactions in Physician Prescription Behavior: The Role of Opinion Leaders. Journal of Marketing Research 47(5), 883895.

Newman, N. (2013). The Cost of Lost Privacy: Search, Antitrust and the Economics of the Control of User Data. SSRN Working Paper.

Owen, J. and R. Rabinovitch (1983). On the Class of Elliptical Distributions and Their Applications to the Theory of Portfolio Choice. Journal of Finance 38(3), 745-752.

Probst, F., L. Grosswiele, and R. Pfleger (2013). Who will lead and who will follow: Identifying Influential Users in Online Social Networks. Business \& Information Systems Engineering 5(3), $179-193$.

Sákovics, J. and J. Steiner (2012). Who Matters in Coordination Problems? American Economic Review 102(7), 3439-3461.

Sundararajan, A. (2007). Local Network Effects and Complex Network Structure. The BE Journal of Theoretical Economics 7(1).

Tucker, C. (2008). Identifying Formal and Informal Influence in Technology Adoption with Network Externalities. Management Science 54(12), 2024-2038.

Van Mieghem, J. A. (1995). Multi-resource Investment Strategies under Uncertainty. Unpublished doctoral dissertation. Stanford University.

Wormald, N. C. (1999). Models of Random Regular Graphs. In J. Lamb and D. Preece (Eds.), Surveys in Combinatorics, Volume 276, pp. 239-298. Cambridge University Press. 